\title{
"ABRAXAS" A CAFARNAO. I. ANTICIPAZIONI SUGLI AMULETI NEL DEPOSITO SOTTO LA PAVIMENTAZIONE DELLA SINAGOGA
}

\author{
Ermano A. Arslan \\ Accademia Nazionale dei Lincei, Roma \\ Categoria Archeologia \\ ermannoarslan516@gmail.com
}

UDK / UDC: 904:737.1(569.4 Kafarnaum) Izvorni znanstveni rad / Original scientific paper

https://doi.org/10.52064/vamz.54.1.30

Tra i 20.003 esemplari del deposito di moneta divisionale in bronzo, verosimilmente chiuso alla fine del 5 secolo a. C., recuperato sotto la pavimentazione della Sinagoga di Cafarnao, un esemplare, il n. 3766, con foro per la sospensione, si presenta rilavorato a niello come amuleto. Su un lato presenta la scritta in greco $\in \mathrm{V} \Pi$ / ЄПтЄ. Sull'altro lato propone una figura di difficile interpretazione, vista in questa sede come collegata all'immagine indicata come Abraxas sulle "gemme magiche". Un riesame, ancora in corso, del resto del complesso ha rivelato come un'alta percentuale

Le parole-chiave:

Abraxas, Chnoubis, Arpocrate, Amuleto, Gemme magiche, Gnosis, Sinagoga di Cafarnao

II 6 luglio 1972, nel cantiere di scavo, restauro e anastilosi della "Sinagoga" di Cafarnao (Fig. 1-2), in Galilea, sulla riva occidentale del Mare di Tiberiade, diretto da Padre Virginio Corbo OFM e Stanislao Loffreda OFM, veniva rimossa una lastra della pavimentazione nell'angolo N-E del cortile ad Est della Sala di Preghiera (Fig. 3), creando la Trincea XII, successivamente nella planimetria generale dello scavo indicata come Trincea 8012. La struttura del cortile è addossata alla fabbrica della Sala, che è quindi precedente, e viene datata dagli scavatori al 5 secolo, pavimentazione compresa. ${ }^{1}$ Nella preparazione per la posa della lastra rimossa delle monete recuperate, ufficiali o di imitazione, porti tracce indiscutibili di riutilizzo come amuleto, con la collocazione sui tipi di un patrimonio di icone ben noto dalle gemme magiche, con precisi riferimenti all'ambito escatologico egizio. Le raffigurazioni sono incise, ricavate ad impressione e forse smaltate. II contributo si propone come segnalazione preliminare, in una serie di interventi su una tematica molto articolata, collocati in altre sedi e in corso di approfondimento.

(Fig. 4), così come delle lastre adiacenti, vennero recuperati, impastati nella malta, ca seimila tondelli bronzei, tutti interpretati come monete, di cui ebbi notizia nel $1994 .{ }^{2}$ Altri esemplari simili vennero recuperati nella medesima Trincea XII nel 1975, fino a raggiungere il numero di 20363. Rimandando ai numerosi contributi relativi allo scavo della "Sinagoga" e riferendomi al solo recupero dei tondelli bronzei, ricordo come il sollevamento della pavimentazione sia avvenuto anche in altri settori del Cortile e della Sala di preghiera, con trincee di scavo che ne restituirono altri: 220 e 1795 nella XIV, 511 nella XVIII, 67 nella XVII-navata Est,
$1 \quad$ Per la bibliografia, alla quale rimandavo negli anni 1995-1996 (Arslan 1997), relativa alla complessa problematica dell'inquadramento storico-archeologico dell'edificio e alle circostanze del ritrovamento, cfr. Tsafrir 1995, passim. Secondo lo studioso l'edificio, da datarsi al 3 secolo d. C., nel 4 secolo sarebbe stato in funzione ed avrebbe subito solo danni secondari in occasione di un terremoto del $363 \mathrm{~d}$. C. Per tali ipotesi, che appaiono a chi scrive non del tutto convincenti, si rimanda alla lettura dello scavo e delle strutture di Stanislao Lof freda (Loffreda 2005). Le evidenze proposte in questa sede modificano sensibilmente i termini del problema, che andranno sicuramente reimpostati in futuro 


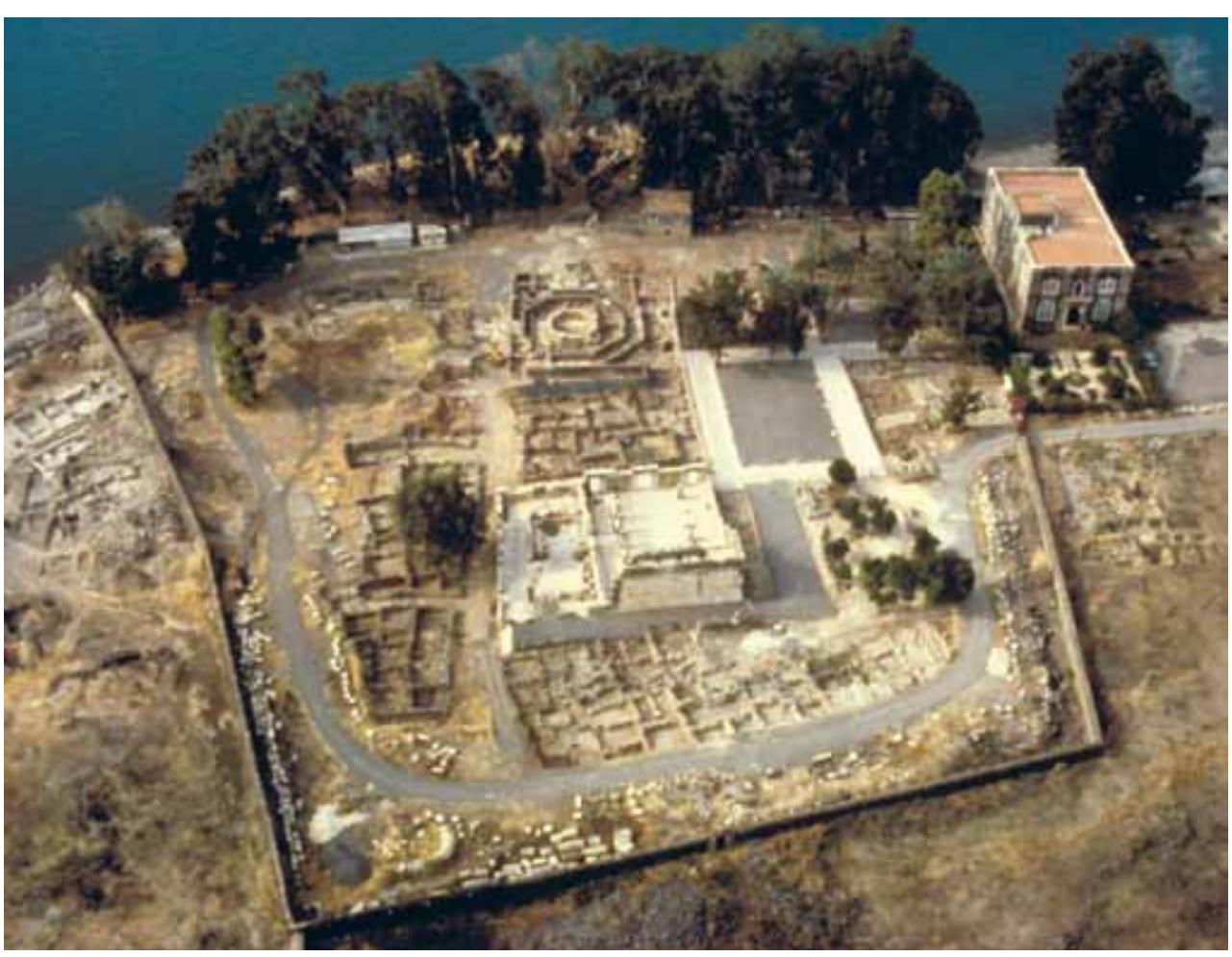

FIGURA 1. Cafarnao. Veduta aerea prima della costruzione della chiesa sulla casa di Pietro (foto Custodia di Terrasanta).

17 nella XVII-navata West, 30 nella XXV (con ulteriori pochi che non è stato ancora possibile conteggiare), per un totale dalla Sinagoga di 23003 esemplari (Fig. 5). I nuclei recuperati vennero tenuti distinti. In un solo caso, nella Trincea XIV-Tesoro, furono selezionati gli esemplari leggibili e scartati quelli apparentemente illeggibili, ora irreperibili.

Il complesso dei tondelli, per il quale non mi risulta sia stata effettuata una rilevazione stratigrafica della giacitura, era costituito in parte da monete, coniate o fuse, spesso con interventi riconoscibili per il riuso come amuleti, ${ }^{3}$ in parte, in percentuale minore, da veri e propri amuleti monetiformi. La moneta, sia quella senza interventi, sia quella con interventi per l'utilizzo per il riuso, era quasi esclusivamente enea ${ }^{4}$ ed emessa dal 3-2 secolo a. C. alla fine del $5 \mathrm{~d}$. C., con le prime serie di Zenone, con una distribuzione regolare nel tempo. Le monete, sia quelle modificate, sia quelle senza apparenti modifiche, prelevate dalla circolazione, vennero collocate singolarmente, a formare un deposito, a partire dalla metà del 4 secolo o poco dopo. Vennero accumulate forse in luogo diverso da quello del ritrovamento, con, dalla fine del 5 secolo, solo episodiche aggiunte di pochissimi esemplari protobizantini e Omayyadi. Infine, il complesso venne occultato, in epoca imprecisata, sotto la pavimentazione del cortile della
Sinagoga, che potrebbe essere sensibilmente più tarda dell'edificio. ${ }^{5}$

I materiali, già fortemente danneggiati dalla permanenza in strati ricchi di calce e settorialmente umidi, vennero puliti in termini molto energici, in bagni con una soluzione fortemente acida, che ne abbassò uniformemente la superficie, specie nelle fragili emissioni imitative di 5 secolo con lega scadente, rimuovendo anche tutto ciò che appariva concrezione calcarea e che invece risultò successivamente essere un rivestimento intenzionale in materiale diverso, utilizzato per collocare tipi derivati dal reimpiego come amuleti.

Questi interventi pregiudicarono fortemente le possibilità di lettura dei materiali, moltiplicando esponenzialmente gli esemplari realmente illeggibili e impedendo, in molti casi per sempre, anche solo la percezione delle specificità di oggetti trasformati in gettoni legati a pratiche magico-religiose. ${ }^{6}$

La conservazione di gran parte dei materiali sotto gli ampi settori di pavimentazione rimasta intatta potrebbe permettere in futuro uno scavo stratigrafico corretto, per il recupero di un complesso, che per ora rappresenta un unicum, e che, difeso dal-
3 Per la definizione di una "Dimensions of a Category Magic" e, nello specifico, di "amuleto", classe monumentale con funzioni e significati estremamente complessi e costantemente sfuggenti, cfr. Frankfurter 2019, passim e specie 24.

4 Si ha qualche Antoniniano di 3 secolo, in argento povero.
$5 \quad$ Il terminus ante quem non parrebbe indicato dalla moneta Omayyade. Non escludo però la penetrazione di monete tarde negli interstizi della pavimentazione, non rilevata al momento dello scavo.

6 Sulla magia nel mondo antico cfr. Frankfurter 2019 a e gran parte dei testi citati in questo contributo. 
Figura 2. Cafarnao. Cortile della Sinagoga (foto Custodia di Terrasanta).

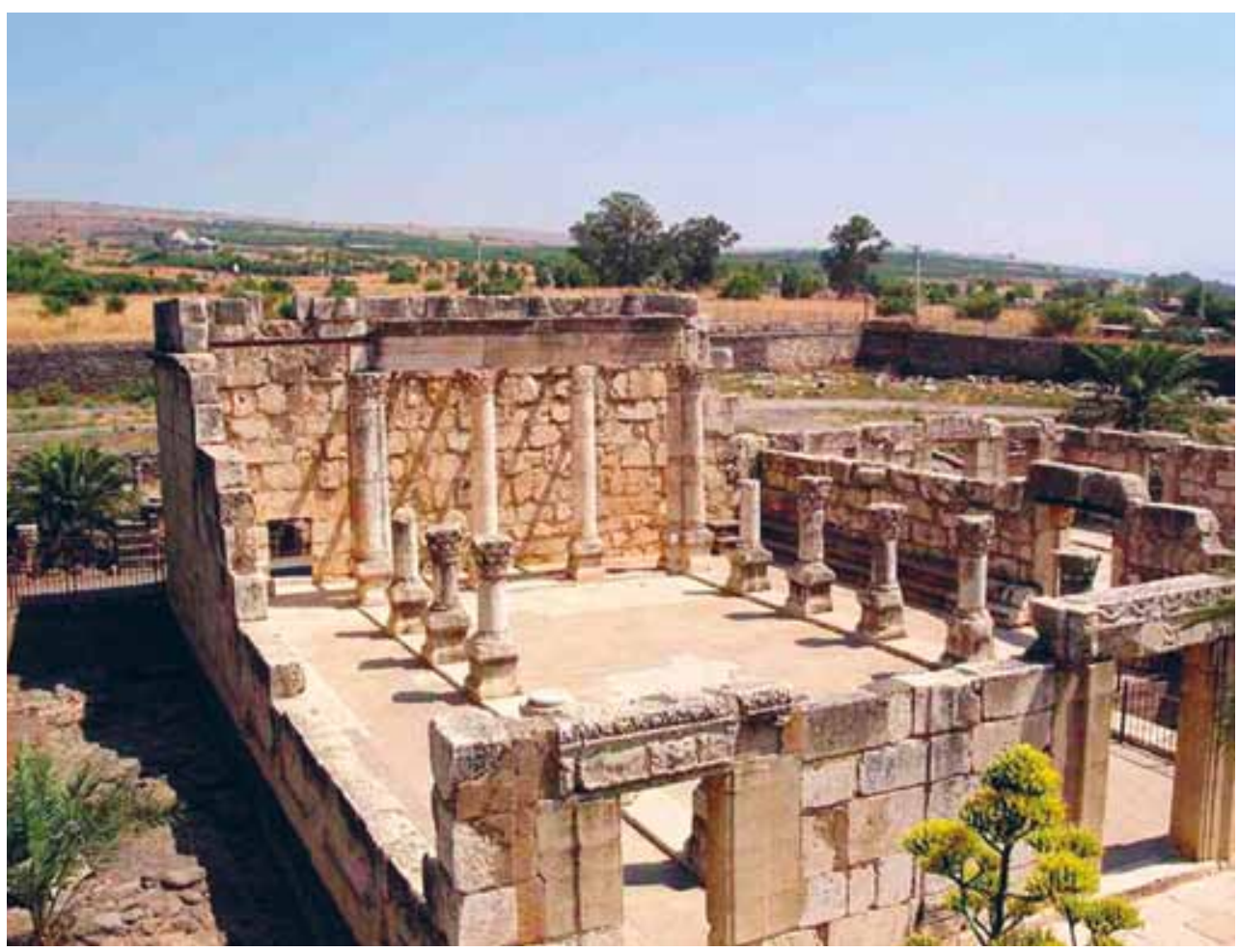

la pavimentazione sovrapposta, dovrebbe essere in migliori condizioni, da trattare con un restauro capace di risparmiare le fragilissime tracce del reimpiego della moneta come amuleto, con la possibilità di analisi a tutti livelli. E sia come numero e qualità dei documenti che come contesto di conservazione.

Lo studio del deposito, inteso come esclusivamente monetario, venne iniziato già con i primi recuperi da August Spijkerman OFM, ${ }^{7}$ con la suddivisione dei materiali in grandi classi tipologiche e per autorità emittente, in termini catalogici tradizionali. Non venne portato a termine, per la Sua prematura scomparsa nel 1973. Nel 1995 chi scrive prese contatto con Padre Loffreda OFM, ottenendo di venire ospitato a Gerusalemme presso la $\mathrm{Cu}$ stodia di Terrasanta e di venire incaricato di studiare il deposito. ${ }^{8}$ I lavori si prolungarono fino al 22 luglio del 2012, con la fondamentale collaborazione operativa di Bruno Callegher, al quale pure va il mio ringraziamento, che operò anche in autonomia sulle monete di ritrovamento isolato dal centro urbano di $\mathrm{Ca}$ farnao e su altri complessi conservati presso la Custodia. ${ }^{9}$ Bruno Callegher ebbe anche modo di analizzare e pubblicare le classi con tipi imitativi di fantasia del deposito. ${ }^{10}$

Chi scrive procedette, sempre in collaborazione con Bruno Callegher, alla numerazione inventariale di tutto il complesso, con imbustamento singolo e rilevazione del peso al centigrammo, completandone la scansione con risoluzione 1200. Compilò sinteticamente la scheda inventariale di tutte le monete delle trincee minori e di ca 4100 esemplari della trincea 12 , senza la registrazione del riuso generalizzato delle monete come gettoniamuleto, allora non ancora percepito, ${ }^{11}$ e con una distinzione solo sommaria per il 5 secolo tra le monete enee di emissione ufficiale e le monete imitative, coniate o fuse. ${ }^{12}$
7 Padre Spijkerman, profondo conoscitore della monetazione antica di area siro-palestinese, concluse un primo studio delle monete dalla città (Spijkerman 1975), con l'esame di 766 monete. Le monete della città sono state nuovamente studiate e pubblicate da Callegher 2007.

8 Sono immensamente grato a Stanislao Loffreda e a tutti i frati della Custodia di Terrasanta, che mi accolsero per mesi nella loro comunità, con fraterna amicizia, permettendomi di vivere una straordinaria esperienza spirituale ed umana ancor prima che scientifica.
9 Callegher 1997; Callegher 2007

10 Callegher 2016.

11 Ciò implica l'inaffidabilità dei dati statistici proponibili per gran parte de gli esemplari, sia ufficiali che imitativi (coniati e fusi) emessi dalla fine del 4 alla fine del 5 secolo, che hanno subito un riuso come gettoni che ne ha modificato radicalmente la natura. Ad essi i criteri e gli strumenti utilizzati per la schedatura numismatica tradizionale risultano inapplicabili e andranno discussi e for malizzati in altro modo in futuro.

12 La distinzione tra prodotti di zecca di scarsa qualità e prodotti imitativi di buona qualità è problema comune, spesso senza soluzione, a tutti coloro che studiano moneta divisionale enea. Essa rende insidiosa la griglia tipologica proposta anche nelle sedi più autorevoli, come RIC 10. La produzione, in un luogo determinato, di monete imitative con l'indicazione in esergo di una zecca anche molto lontana, o di altra epoca, inficia spesso i calcoli statistici relativi alla mobilità nello spazio della moneta, fondamentale per le analisi storico economiche. Le statistiche proposte in Arslan 1997, relativamente al 5 secolo, vanno quindi utilizzate con grande prudenza. 


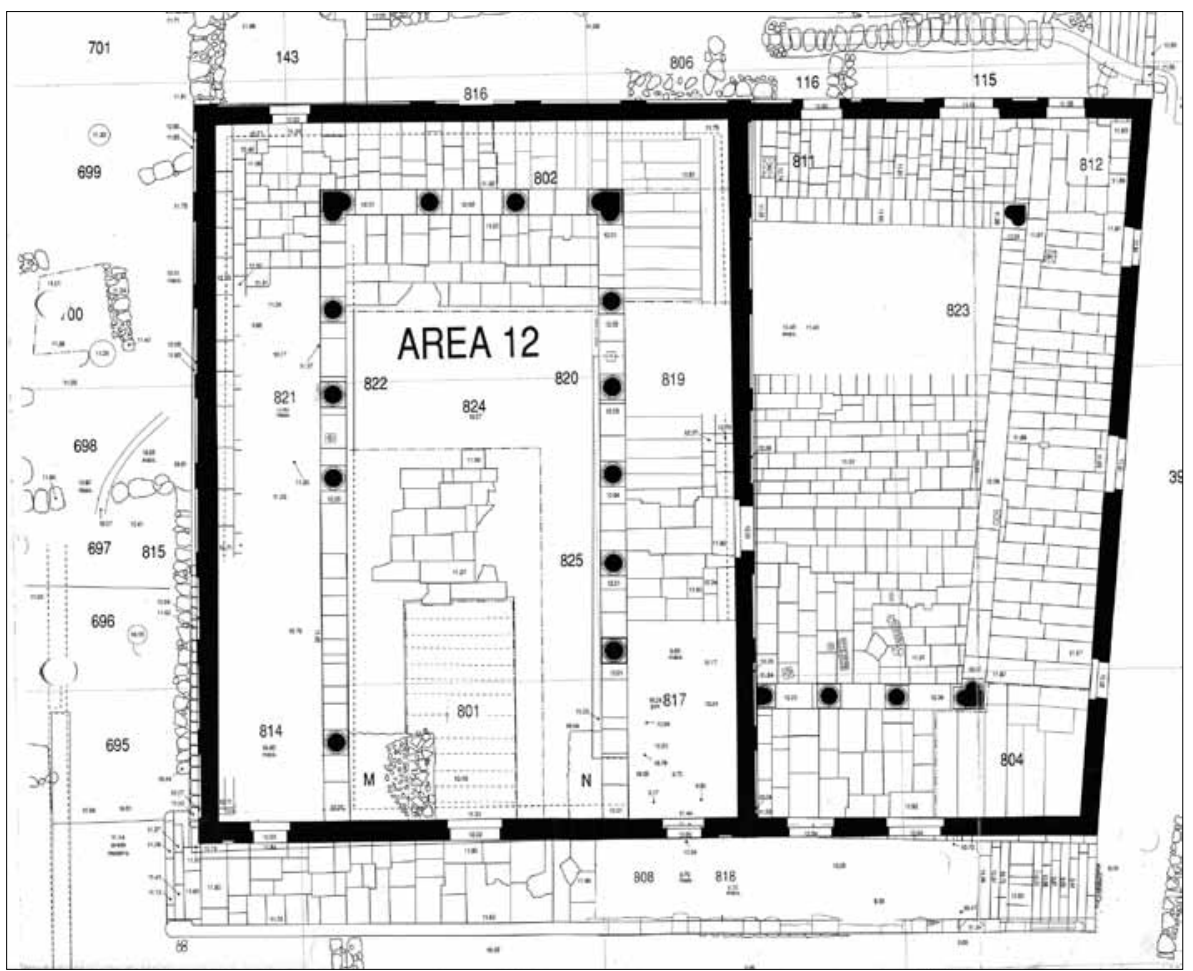

Figura 3. Cafarnao. Planimetria della Sinagoga con indicazione de settori scavati (foto S. Loffreda).

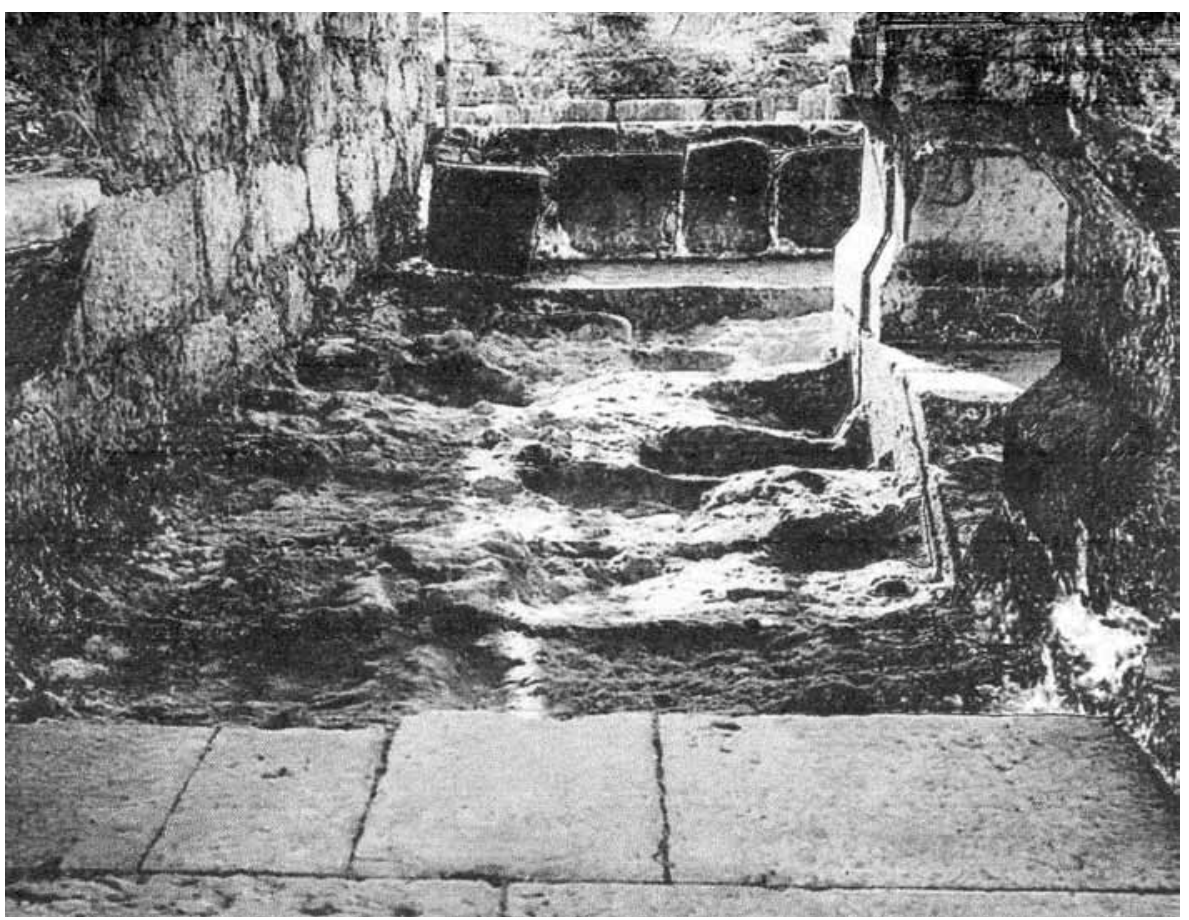

Figura 4. Cafarnao. Preparazione per la posa in opera della pavimentazione della Sinagoga (da Loffreda 2005).

In attesa della conclusione della schedatura di tutto il complesso, che segnalai nelle sedi opportune, ne isolai un campione statistico casuale del $15 \%$ (3058 monete, di cui 1925 giudicate leggibili), 13 che elaborai per definirne sia l'arco cronologico coperto, dalla moneta tolemaica di 2-1 secolo a. C. a quella di Zenone, primo regno, che la meccanica di formazione, con deposizione distribuita nel tempo di singoli esemplari, ${ }^{14}$ che per impostare l'analisi della struttura della massa circolante cui si era attinto, con la data presunta di inizio dell'accumulo e con il calcolo delle percentuali di presenza delle varie zecche tardo-antiche, da collocare nel quadro complessivo della mobilità della moneta di terzo livello nel Mediterraneo orientale. 
FiguRA 5. Cafarnao. Il deposito di monete-amuleto della Sinagoga nella prima fase del recupero (foto S. Loffreda).

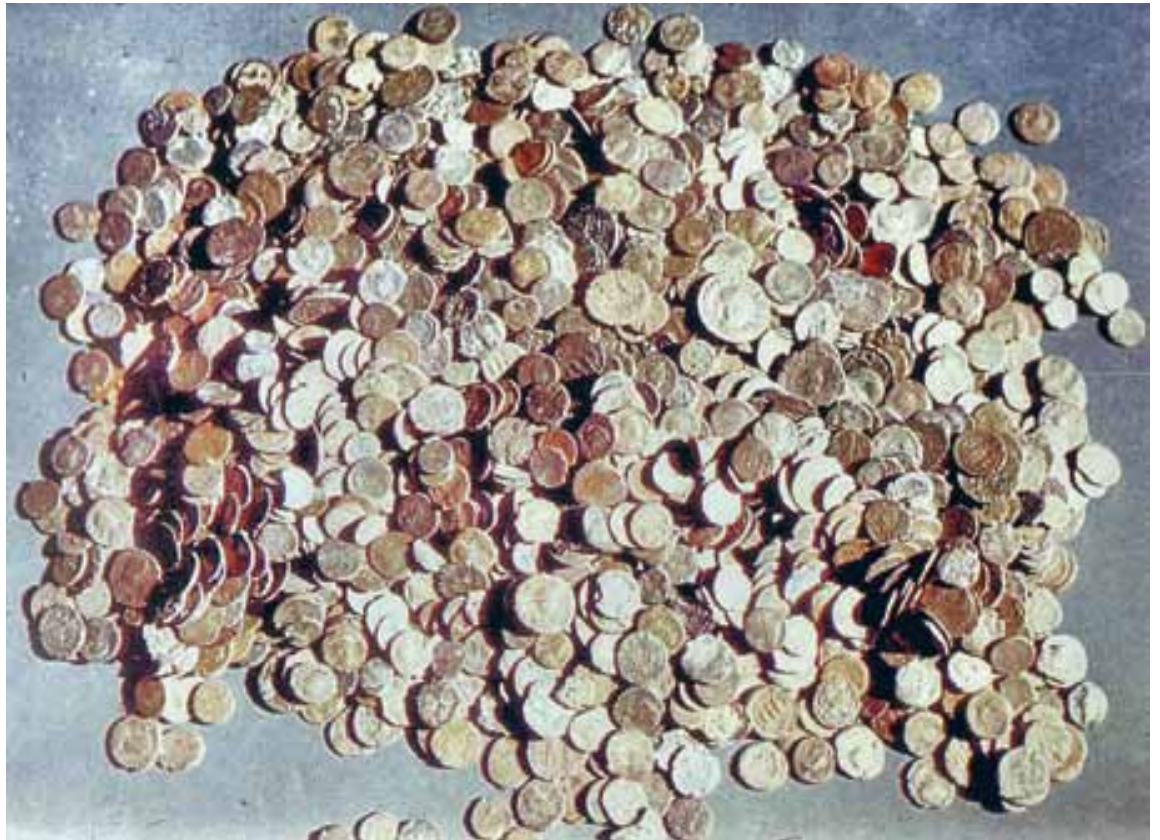

Parallelamente isolai e segnalai alcune presenze fortemente minoritarie ma di rilevante importanza culturale ed ideologica. Come la moneta isiaca, con la proposta dell'emissione anche ad Alessandria, ${ }^{15}$ e la moneta imitativa di tipo Axumita, ${ }^{16}$ presente nel 5 secolo in esemplari fusi di standard AE $5 .{ }^{17}$

Affrontai anche l'analisi dell'evoluzione ponderale del cd. Num$m u s^{18}$ e dei tempi di resistenza in circolazione delle emissioni ellenistiche e romane imperiali. ${ }^{19}$

In alcuni di questi programmi di studio sottovalutai la presenza della moneta divisionale imitativa di produzione locale, praticamente maggioritaria nel 5 secolo. Essi mi appaiono oggi non utilizzabili, se non con grande prudenza, per ipotesi relative alla mobilità della moneta sul territorio, nel 5 secolo, dalle zecche ai mercati. $^{20}$

Tema, questo, ulteriormente reso più complesso dal fenomeno del riuso della moneta, coniata e fusa, ufficiale e imitativa, per la trasformazione in gettone-amuleto, prodotto di diverso significato e funzione, che è alla base di questo contributo.

\footnotetext{
15 Arslan 1996a; Arslan 2003a

16 Arslan 1996c, per le imitazioni miniaturizzate fuse di monete axumite e romane imperiali della seconda metà del 5 secolo e precedenti.

17 Arslan 1996b. Ho adottato nei miei più recenti contributi con analisi di emissioni enee, ufficiali o imitative, di 5-4 secolo, la definizione convenzionale di AE5, per il divisionale (probabilmente 1/3) del cd. Nummus, definito convenzionalmente come AE4 O AE IV, con standard ponderale teorico riferito allo Scrupulum (1/24 di uncia, circa $1.135 \mathrm{~g})$.

18 Arslan 2003b.

19 Progetti in parte abbandonati per alcuni anni, a causa di un forzato allontanamento da Israele.

20 Il difficile controllo della problematica della produzione locale della moneta divisionale enea, di terzo livello, indebolisce le conclusioni anche di alcuni dei contributi proposti nell'incontro su Production und Recyceln del 2016 (cfr. recensione in Arslan 2020b).
}

Il complesso di 23.003 oggetti monetiformi recuperati, con scansioni ormai completate ma solo settorialmente riesaminate negli ultimi mesi (al $30 \%$ ca.), in gran parte ha rivelato, ad un esame attento e con forte ingrandimento, essere costituito da monete, ufficiali o imitative, con tracce, talvolta molto labili ma comunque riconoscibili (con fatica), di interventi meccanici sui tipi, per modificarli o sostituirli. Il resto è costituito da tondelli monetiformi prodotti ad hoc per fusione, con tipi funzionali all'utilizzo come "amuleto" e da monete, ufficiali o imitative, senza tracce di modifica intenzionale, che possono essere scomparse a causa della maldestra pulizia al momento dello scavo. ${ }^{21}$

Il complesso di Cafarnao può quindi essere inteso come un deposito di amuleti monetiformi e propone tematiche articolatissime, alcune del tutto inedite, per le quali mi è parso privo di senso tentare di giungere a ipotesi e tanto meno a conclusioni, se non molto preliminari, prima di aver sufficientemente sviluppato un impegno di ricerca prevedibilmente di anni, se non di lustri. Tale inoltre da non poter essere sviluppato da chi scrive, per ovvie ragioni anagrafiche e l'inadeguatezza a trattare tematiche in molti casi estranee alla sua esperienza professionale. Mi limito quindi, per ora, a proporre alcuni dati preliminari di anticipazione in questo contributo, indispensabili per un primo inquadramento della problematica proposta dal deposito di Cafarnao, in omaggio al collega ed amico Željko Demo, impegnandomi nel contempo a scrivere altri due contributi, sempre di anticipazione, su ulteriori aspetti specifici del complesso, per dedicarli in un secondo tempo a due figure che mi sono particolarmente care, per le quali sono state promosse raccolte miscellanee di scritti di colleghi e amici. Si tratta di Maria Caccamo Caltabiano, in occasione della conclusione del suo impegno universitario a Messina,

21 Con solo il $30 \%$ degli esemplari analizzati, non è ancora possibile valutare percentualmente la presenza delle diverse classi. 
e dell'archeologa milanese Maria Teresa Grassi, recentemente e prematuramente scomparsa.

Alcuni dei temi assenti in questo contributo, o ai quali ho dedicato solo cenni preliminari, saranno affrontati in queste due sedi.

Lo studio esaustivo e l'edizione dei materiali di Cafarnao saranno certo affrontati in futuro da un'équipe di ricercatori attivi in diversi ambiti disciplinari coinvolti, con adeguata strumentazione tecnica, quando il complesso, ${ }^{22}$ conservato presso lo Studium Biblicum Franciscanum a Gerusalemme, sarà di nuovo facilmente accessibile.

Nel materiale fiora recuperato sono risultati assenti esemplari monetiformi con appiccagnolo e sono stati registrati solo pochissimi isolati esemplari con foro marginale per la sospensione. ${ }^{23}$ I materiali raccolti nel deposito non erano quindi prodotti per essere utilizzati come "pendenti" ed "indossati", se non in casi eccezionali. Non erano destinati ad essere osservati dall'esterno, come di norma i "pendenti", che sono sempre utilizzati a stabilire una comunicazione tra chi di indossa e chi li osserva. Quindi dovevano essere collocati in qualche contenitore, se in qualche modo conservati, oppure erano destinati a raggiungere immediatamente il deposito come offerta.

Tra i pochi esemplari con foro presenti nel complesso si differenzia formalmente e strutturalmente il n. 3766, di gr. 1.81, con diam. $15.5 \times 14.5 \mathrm{~mm}^{24}$ e con assi del riuso a $180^{\circ},{ }^{25}$ che risulta essere una moneta reimpiegata, non classificabile, con lievi tracce dei tipi originari. Sul lato A si ha una figurazione a prima vista incomprensibile e sul lato B una leggenda in lettere greche su due righe ${ }^{26}$ con andamento da sinistra a destra, ${ }^{27}$ ottenute operando un'incisione con uno strumento metallico appuntito, successivamente riempita con un materiale indeterminato di colore più scuro, ${ }^{28}$ quasi del tutto caduto sul lato $A$, dove è visibile l'incisione del metallo della moneta.

Il foro è collocato in posizione tale da permettere, in sospensione, la lettura della leggenda greca.

22 Sotto la pavimentazione della Sinagoga di Cafarnao, solo parzialmente rimossa, giacciono probabilmente altri consistenti nuclei di monete-amuleto, forse di centinaia di migliaia.

23 Per ora i nn. 3766, 11034, 11619, 12326, 12758, 17298, 17321. Per le monete con foro, per servire da pendente, o amuleto, o talismano, cfr. Perassi 2011a; Perassi 2011b; Doyen 2013.

24 II foro mostra di aver ceduto in antico. Questo può aver giustificato l'abbandono nel deposito.

25 L'indicazione di D/ e di R/ è del tutto convenzionale.

26 Sembrerebbe presente in basso anche una terza riga, con solo una A leggibile. Per l'uso della scrittura nelle pratiche magiche cfr. Frankfurter 2019c.

27 La direzione della scrittura, non speculare, è la medesima utilizzata nelle gemme magiche (Dasen, Nagy 2019, 420) ed esclude un uso sigillare del manufatto

28 La tecnica utilizzata parrebbe essere il "niello", da "nigellum", documentata da epoca molto remota, anche nell'antico Egitto. La lega metallica di colore scuro posta a riempire l'incisione include zolfo, rame, argento e spesso anche piombo.

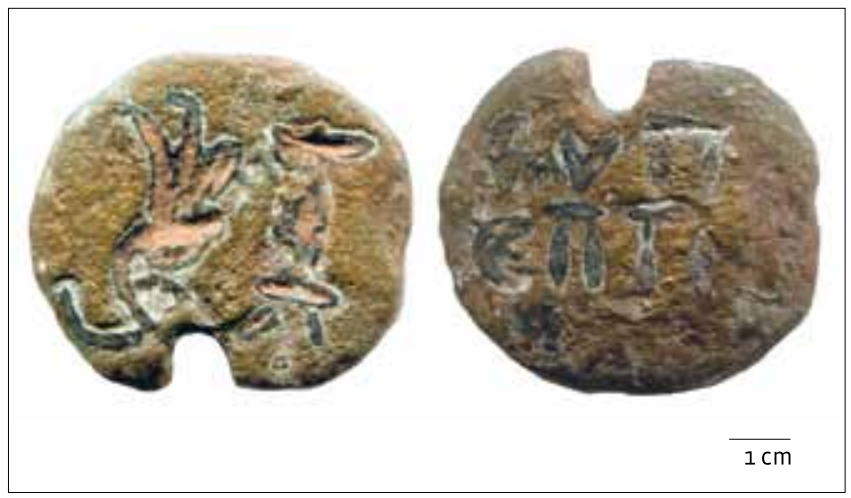

FIGURA 6. Deposito della Sinagoga di Cafarnao, n. 3766, gr. 1.81 (foto E. A. Arslan)

La moneta-amuleto n. 3766, unicum nel deposito di Cafarnao, rimane priva di confronti tra gli amuleti e i pendenti romani e tardo-romani a me finora noti.

La leggenda del lato $B, € V \Pi$ / $€ \Pi T \epsilon$, in lettere maiuscole greche, è riferita ad aspetti relativi a funzioni digestive, ${ }^{29} \mathrm{ma}$ attende la verifica dell'epigrafista o di un conoscitore delle funzioni magicoreligiose-taumaturgiche degli amuleti nella tarda antichità.

La figurazione sul lato A mi rimase per lungo tempo del tutto incomprensibile, non ostante la completezza e la facile leggibilità, fino a quando mi venne proposto ${ }^{30}$ di leggerla come la rappresentazione fortemente stilizzata di un tipo iconografico noto dalle cosiddette "gemme magiche" ${ }^{31}$ e indicato con il nome di

29 In una terza riga forse si legge, deformata per lo spazio ridotto, la scritta $[1] A \Omega$. Le leggende sulle gemme magiche sembrano spesso riferirsi alle figurazioni presenti sull'altra faccia del prodotto. Potrebbero essere collegate a momenti del rituale con l'uso della voce, per formule o invocazioni (Dasen, Nagy 2019, 419-420). Non propongo la riproduzione grafica dei materiali di Cafarnao per la conservazione degli esemplari che costringerebbe ad interpretazioni troppo soggettive e a fraintendimenti di quanto è spesso troppo incerto. Non escludo che lo si possa fare in futuro, proponendo diverse possibilità di integrazione per ogni pezzo

30 Devo ringraziare la Prof. Agnes Bencze, storica dell'arte antica all'Università Pázmány di Budapest, che mi ha segnalato The Campbell Bonner Magical Gems Database di Budapest (http://cbd.mfab.hu/) e che ha ampiamente discusso con me gli aspetti stilistici della figurazione. Ringrazio anche gli amici Marco Garzonio, Francesco d'Andria, Christian Orsenigo e i "referenti anonimi" con i quali ho avviato una utilissima discussione sul tema sviluppato in queste pagine.

31 Sulle gemme magiche Bonner 1950, con successiva bibliografia fino a Dagen, Nagy 2019; Magical Gems 2019. Per “Abrasax” (Endreffy, Nagy 2020). Dati probabilmente utili sono stati presentati in Ancient Greek, Roman and Byzantine engraved gems, trasmesso in Zoom nel 2021 (non vidi). Non se ne conosce l'uso in antico, comunque da intendere in contesti rituali (Dagen, Nagy 2019, 434-436). Il deposito di Cafarnao propone una situazione finora inedita e quindi con tematiche assenti in bibliografia. È opportuno utilizzare il termine "gemme magiche", che si riferisce all'indiscutibile connessione di questi documenti a pratiche "magiche", in ogni loro possibile articolazione di significato, a preferenza del termine "gemme gnostiche", che si riferisce ad un complesso ambito ideologico, filosofico, religioso, nel quale non tutti i prodotti, nel tempo portati a costituire questa "classe" monumentale, sembrano inserirsi, se non spesso solo per la natura preziosa del supporto. Alla funzione magica fa riferimento la definizione del Campbell Bonner Magical Gems Database di Budapest, nello spirito di Bonner 1950. Per tali tematiche è sempre fondamentale Jonas 1958 Per la magia in generale cfr. il recentissimo Frankfurter 2019. 


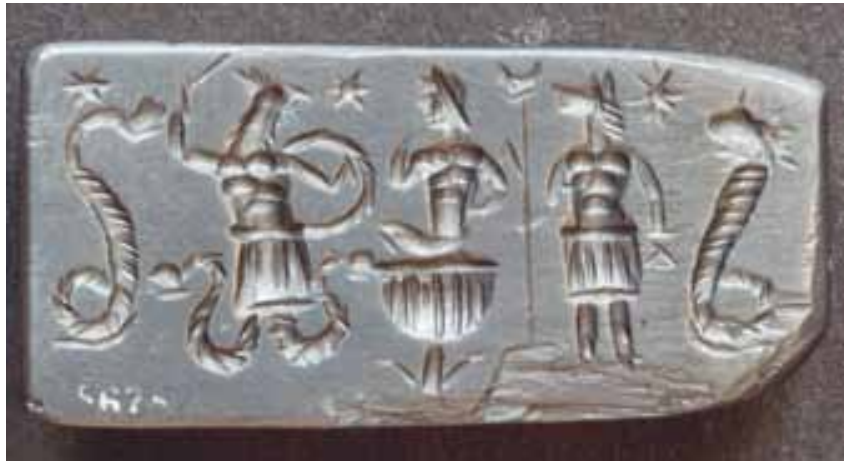

FIGURA 7. Gemma in ematite. Londra, British Museum (da CBd-949).

"Abraxas" (o Arbasax-Abrasax). Nelle gemme appare ${ }^{22}$ quasi esclusivamente in una formulazione "canonica", loricata, con testa di gallo crestato e gambe anguiformi, scudo rotondo, spesso iscritto, con flagello alzato.

Ne abbiamo un'esemplificazione in una "gemma" in ematite del British Museum ${ }^{33}$ (cbd.mfab.hu/cbd/949), di forma anomala, nella quale l'immagine anguipede si presenta affiancata da altre quattro icone (Fig. 7), 34 le più frequenti sulle "gemme magiche", certamente le figure più importanti nella concezione "gnostica", o di ispirazione "gnostica", alla quale tradizionalmente vengono riferite le gemme. In esse certamente, distinguendole dalle gemme sigillari e da quelle da riconoscere come oggetto prezioso decorato, ${ }^{35}$ vanno riconosciuti oggetti rituali, o meglio amuleti, da riferire a un ambito ideologico e religioso a mio avviso non unitario, ma articolato in percorsi differenziati, pur con evidenti premesse comuni.

Nella gemma londinese appaiono in sequenza, ${ }^{36}$ forse in collocazione gerarchica, da d. a s. Uraeus,,$^{37}$ Abraxas, ${ }^{38}$ Horus/Arpocrate sul fiore di loto, ${ }^{39}$ entità probabilmente centrale, Anubis, Chnoubis. $^{40}$

L'amuleto n. 3766, chiaramente da collegare alla classe delle "gemme magiche" per la presenza e l'impaginazione della leggen$\mathrm{da}$, ne è però dissimile per il materiale, ${ }^{41}$ il bronzo, rarissimamente utilizzato, e la tecnica a niello usata per rendere visibili le lettere della leggenda. La figurazione, infine, appare molto diversa e obiettivamente di non facile interpretazione; non sembra, a mio avviso, derivare dal modello iconografico alla base dell'immagine comunemente presente sulle gemme magiche, ma potrebbe essere una elaborazione, fortemente "astratta", dell'immagine virtuale originaria, forse descritta in testi scritti, o tramandata oralmente, e quindi non necessariamente figurativa..$^{42}$

Ad una analisi stilistica preliminare l'immagine della faccia A presenta obiettivamente pochi aspetti comuni con la maggior parte delle gemme. L'immagine forse di Abraxas è riconoscibile solo per pochi, ma essenziali, elementi dell'incisione, a malapena riconoscibili, come la cresta di gallo e le gambe anguiformi. Si coglie un'elaborazione di stilemi originalissimi, riferibili forse ad un archetipo, a noi non noto, tendenzialmente ben distinto dallo schema iconografico alla base della quasi totalità delle immagini riferite ad "Abraxas", per la leggenda o per analogia, presenti nel Database di Budapest e nella Sylloge Gemmarum Gnosticarum. ${ }^{43}$
32 Cosentino 2013, Nagy 2018, con il riconoscimento come moderno del bronzetto di Avenches, propone una chiara e sintetica analisi della fortuna di questa tematica nella moderna cultura europea. Vi si sottolinea anche come manchino riferimenti all'immagine di "Abraxas", così come al suo nome, nelle fonti scritte greco-romane (cfr. Vitellozzi 2018), invece molto frequente nella classe monumentale rappresentata dalle gemme, di norma in materiale prezioso, con l'utilizzo di pochi altri supporti di varia natura, forma e materiale e anche episodicamente in piombo o metallo o terracotta. Rare appaiono le provenienze sicure, per un materiale che ha sofferto per secoli di collezionismo e per un interesse moderno collegato all'esoterismo e alla superstizione, che giustificano la presenza di derivazioni e sviluppi iconografici nelle età successive, per i quali cfr. Mastrocinque 2003, come appunto I "Abraxas" di Avenches. Tra questi nei quali vanno molto probabilmente inseriti i medaglioni siciliani con appiccagnolo e la lamina in piombo perduta, segnalati come antichi in Manganaro 1963, di provenienza insicura e forse sei-settecenteschi. Per i ritrovamenti di gemme magiche con la figura anguipede in Italia meridionale e Sicilia, in attesa dei dati raccolti da Campedelli 2019, cfr. Pugliese Carratelli 1953 per gli esemplari della collezione Capialbi di Vibo Valentia. Del tutto sicuro è il grande amuleto ovale in siltite, bifacciale, da Nea Paphos in Cipro. L'amuleto viene di scusso in Sliwa 2013; Alexandra 2015; Mancini 2015; per ultimo in Graham 2021. La complessa composizione nel campo presenta molti contatti con i materiali di Cafarnao, ma anche molte divergenze, che lo collocano in una parallela e diversa dimensione ideologica e rituale. Nei contributi che ho in preparazione su tali temi tenterò di aggiornare la sequenza della documentazione disponibile, in continuo aumento con contributi saggistici e segnalazioni di gemme magiche inedite.

33 Ematite Br. M. G 252 (EA 56252). Nel "The Campbell Bonner Magical Gems Database”, n. CBd-949, attribuita al 3 secolo.

34 Per il significato della presenza di figurazioni sugli amuleti cfr. Frankfurter 2019d.

35 Nella sequenza delle icone presenti sulle gemme, per alcune, specie quelle riferite a miti e divinità di ambito ellenico, si ipotizza un possibile collegamento con la produzione tradizionalmente intesa come gnostica, come anche recentemente proposto.
36 Una sequenza simile, con figure in parte diverse e in ordine differente è anche in una gemma del Museo Nazionale di Archeologia in Lisbona (MNA E540): Veiga 2007, Perea Yébenes 2018. La posizione di Arpocrate, nelle due gemme, sempre centrale, indica la divinità gerarchicamente più importante.

37 Ciampini 2007. Sulle tradizioni cultuali e superstiziose, non solo mediter ranee, vive ancor oggi per i serpenti, sia in senso positivo che negativo, esiste ampia letteratura storica, archeologica, etnografica. Cfr. Di Nola 2001; Giancristofaro 2010; D’Andria 2010; I/ serpente nell'area del Mediterraneo 2010 (specie Spera 2010)

38 Nagy 2018

39 El-Khachab 1971.

40 Su Chnoubis cfr. Mastrocinque 2008; Dasen, Nagy 2012; Dasen, Nagy 2019, 428-455. Sarebbe una figura minore dell'astrologia egizia. Con la classe con l'immagine di "Abraxas" rappresenta il $25 \%$ delle gemme "gnostiche", su più di 1000 esemplari. Non sembra avere funzioni magiche, ma legate alla salute e ai legami amorosi (437).

41 L'analisi della tematica delle gemme magiche in Dasen, Nagy 2019, 416 ap pare circoscritta alla glittica e non cita la classe degli amuleti da riuso di moneta di bronzo, classe comunque ancora sconosciuta alla comunità dei ricercatori

42 Per la faticosa interpretazione dello schema dell'Anguipede come derivato da tradizioni religiose giudaiche cfr. Dasen, Nagy 2019, 426.

43 Sylloge Gemmarum Gnosticarum 2003; Sylloge Gemmarum Gnosticarum 2007. In Nagy 2018 si indica come fossero repertoriati in quell'anno ben seicentosettanta prodotti con l'immagine di "Abraxas”. L'esame degli esemplari noti rivela comunque una serie di "tipi" dell'essere indicato come "Abraxas" molto differenziati, forse non adeguatamente analizzati singolarmente in bibliografia. In futuro sarà necessario un sistematico approccio "storico-artistico" a prodotti, talvolta qualitativamente discontinui, ma anche spesso stilisticamente molto elaborati. 
Immagini che mi paiono espressione di una cultura artistica solo debolmente collegata alla tradizione greco-romana-egizia della grande parte della documentazione nota, e che sono anche presenti nei documenti, che vedremo, nel deposito di Cafarnao. ${ }^{44}$ Saremmo tentati, come ipotesi di lavoro, per le caratteristiche e la collocazione geografica del deposito di Cafarnao, di collocarlo nell'ambito giudaico ellenistico romano egittizzante ${ }^{45}$ del Mediterraneo Orientale, ma i possibili riferimenti all'ambito giudaico appaiono labilissimi, se non assenti, nei materiali del deposito. ${ }^{46}$

Comunque per il riconoscimento del tipo sottolineo come la cresta del gallo sia particolarmente visibile, come lo scudo sia portato a fondersi con un torso quasi inesistente, che tutto si risolve nelle gambe/serpenti, che si sviluppano e si avvolgono come tentacoli, incrociandosi e solo accennando alla cifra a doppio semicerchio, a , caratterizzante e costante nell'immagine dell'Anguipede nelle altre varianti della serie iconica intesa come di "Abraxas".

Le gambe anguiformi giungono a sorreggere, sembrerebbe entrambe, convergendo sulla destra del campo, una figurazione minore, indistinta, che parrebbe poggiare su una base piana, con un copricapo (?) largo e piatto. La nostra icona potrebbe essere una variante stilizzata della figura, solo parzialmente teriomorfa, che troviamo in una gemma a Londra, CBd-629, 47 con un "Abraxas" a testa di gallo non anguipede, con una lunga asta, che sorregge con la mano una figura secondaria, che potrebbe rappresentare Horus/Arpocrate su un alto piedestallo.

La costruzione dell'immagine nell'amuleto n. 3766 appare particolarmente complessa e nel contempo sintetica, giocando nel campo bidimensionale con un'incisione a linee tutte curve, che si intrecciano. Si allontana così da quasi tutti i grafemi antropomorfi e teriomorfi caratterizzanti le formulazioni "naturalistiche" dell'icona canonica, rinunciando ad una riconoscibilità immediata.

Nella documentazione del Database di Budapest affiorano solo alcuni pochi documenti, fortemente minoritari, che linguisticamente e stilisticamente possono essere correlati, con un certo coraggio, all'amuleto di Cafarnao.

44 Nei materiali di Cafarnao, tutti di difficilissima lettura, l'immagine convenzionalmente riconosciuta come "Abraxas" sembra proporsi in innumerevoli varianti, negli esemplari finora esaminati, tutte in qualche modo collegate agli schemi più comuni proposti nelle gemme. Mi riservo di affrontarne una trattazione analitica in futuro, in una sede appropriata, limitandomi in questo contributo ad accennarne solo la presenza.

45 Per il collegamento con l'ambito cultuale egizio cfr. Ciampini 2007; Dasen, Nagy 2019, 425 (riferimenti alla tradizione egiziana anche molto antica), 431432 , viene proposta una produzione delle gemme in una pluralità di centri nel Mediterraneo orientale, ma con diffusione ubiquitaria nel territorio dell'Impero, dall'età ellenistica al 3 secolo d. C. Dielemann 2019; Quack 2019.

$46 \quad$ L'ipotesi di un collegamento stretto con il cristianesimo, che si sviluppa comunque sincronicamente nei medesimi ambiti territoriali e culturali, appare anch'essa ormai molto fragile, anche se affiora episodicamente ancora in alcuni dei contributi in Frankfurter 2019. Appare probabile che, nel caso delle gemme magiche e negli amuleti di Cafarnao, si abbiano forme di contaminazione tra religioni e culture diverse (Dasen, Nagy 2019, 422; Dasen 2019; Mastrocinque 2019).

47 Londra Br. M. n. G 367 (EA 56367). In iscrizione è ı $\alpha \omega|\sigma \alpha \beta| \alpha \omega$.

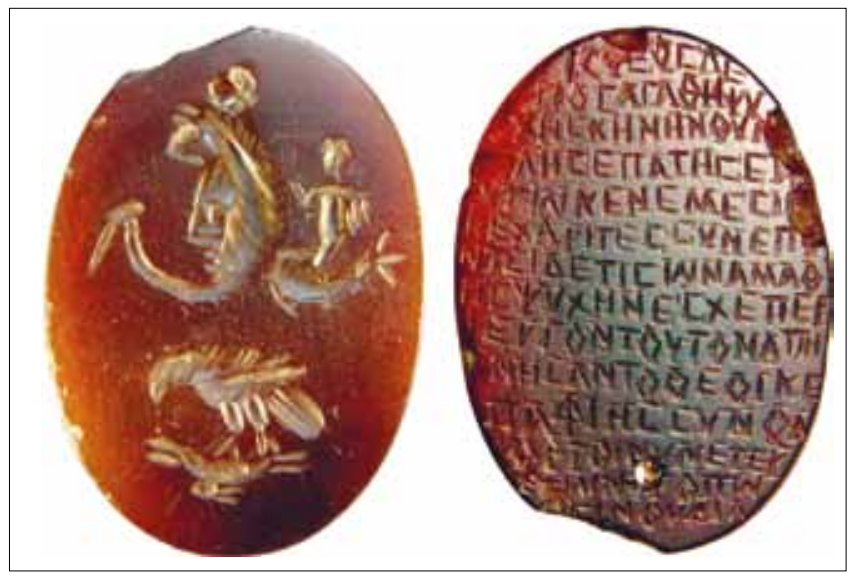

FIGURA 8. Gemma con figura anguipede e Arpocrate. Londra, British Museum. N. MMEu_G 367, EA 56367 (da CBd-3818).

Ad esempio, nella "gemma magica" CBd-3818 del British Museum (Fig. 8), ${ }^{48}$ si percepisce la medesima tensione alla disaggregazione di un prototipo e alla sua riaggregazione in un'immagine ricomposta in una diversa strutturazione organica, lontana sideralmente da ogni condizionamento naturalistico, ma con un fortissimo impatto espressivo.

La costruzione dell'immagine si sviluppa su un percorso di astrazione concettuale analogo nei due prodotti, nella gemma di Londra e nell'ipotetico "Abraxas" dell'amuleto n. 3766, ma con strumenti per l'incisione e con supporti materiali diversi, la pietra dura e il bronzo. Quindi gli esiti figurativi sono divergenti, sempre comunque ad un alto livello di cultura espressiva, ma con premesse forse comuni. Non a caso vedremo più avanti le puntuali analogie stilistiche e costruttive tra la gemma del British Museum e un altro esemplare del deposito, il gettone-amuleto $\mathrm{n}$. 4322 (Fig. 14), che si colloca come trait d'union concettuale tra il pendente bronzeo e la gemma in pietra dura. ${ }^{49}$

Il lato decorato della gemma $\mathrm{CBd}-3818$, propone incise due immagini complesse. Quella in basso è di evidente derivazione ellenistica, con un'aquila che stringe nelle zampe una lepre, con un significato probabilmente comprensibile solo in collegamento con l'icona superiore, priva di qualsiasi confronto figurativo coevo.

\section{CBd-3818 Londra, Br. M. n. MMEu_G 367, EA 56367}

49 La creazione dello schema iconografico dell "Abraxas", estraneo alle tradizioni figurative del Mediterraneo antico, non sembrerebbe quindi limitata all'immagine più comune delle gemme (Dasen, Nagy 2019, 416), ma anche svilupparsi in altra direzione, con la creazione di immagini teriomorfe composite come nella Fig. 19, quasi un ricalco della gemma CBd-3818. 


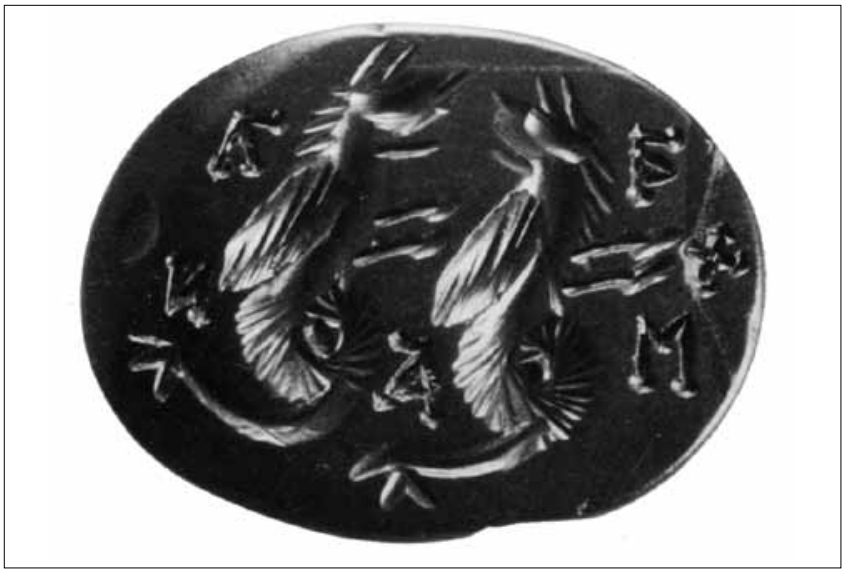

FIGURA 9. Gemma con due anguipedi-lupo alati. Berlin-Aeg_s. n. (da CBd-2129).

La figura, con testa ferina volta a s., è sormontata da una riproduzione miniaturizzata di se stessa, soluzione comune negli amuleti monetiformi magici del deposito, come si vedrà, ed è presentata con un corpo lunato, in parte rigonfio, che sembra privo di braccia, invece accennate in basso in due brevi tratti orizzontali.

Il braccio a destra (è il braccio sinistro della figura) sostiene un minuscolo Horus/Arpocrate, forse con trattini incisi che accennano ad una cornucopia, ben riconoscibile, anche se in una costruzione molto abbreviata e sintetica, ma tale da potenziarlo come visibilità. Nel Database di Budapest, in una lettura diversa dalla mia, viene descritto come "a naked young man ... standing on a fish", interpretando come un pesce la gamba aperta sulla destra e non individuando come il fanciullo sieda su un elemento orizzontale ben distinto dalla gamba, che sopra abbiamo indicato come una delle braccia.

In basso le gambe anguiformi si aprono nei due semicerchi accostati tipici di Abraxas, con un elemento lineare, forse il flagello delle immagini canoniche, collocato all'estremità del semicerchio di sinistra.

La maestria dell'incisore fa tesoro dei pesanti condizionamenti del materiale del supporto, diaspro rosso, durissimo e fragile, che lo costringono a costruire l'immagine ricavando le linee curve da sequenze di brevi incisioni rettilinee, ottenendo l'immagine finale, compatta, plastica e bilanciata, di un insetto mostruoso, che è possibile affiancare a quella dell'amuleto $n$. 3766, ponendo attenzione al fatto che il materiale di quest'ultimo, il bronzo, permetteva un'incisione ben più agevole, che poteva svilupparsi in linee curve.

Tra i rari documenti noti che è possibile inserire in questa ridotta serie di gemme magiche con possibili analogie stilistiche con l'amuleto finora esaminato, $\mathrm{CBd}-3818$, interconnesse da un linguaggio artistico in parte comune, certo anche per consonanze ideologiche e religiose che per ora ci sfuggono, conviene citare l'esemplare di Berlino CBd-2129 (Fig. 9), ancor più significativo per la complessa scelta iconografica, che permette soluzioni vir-

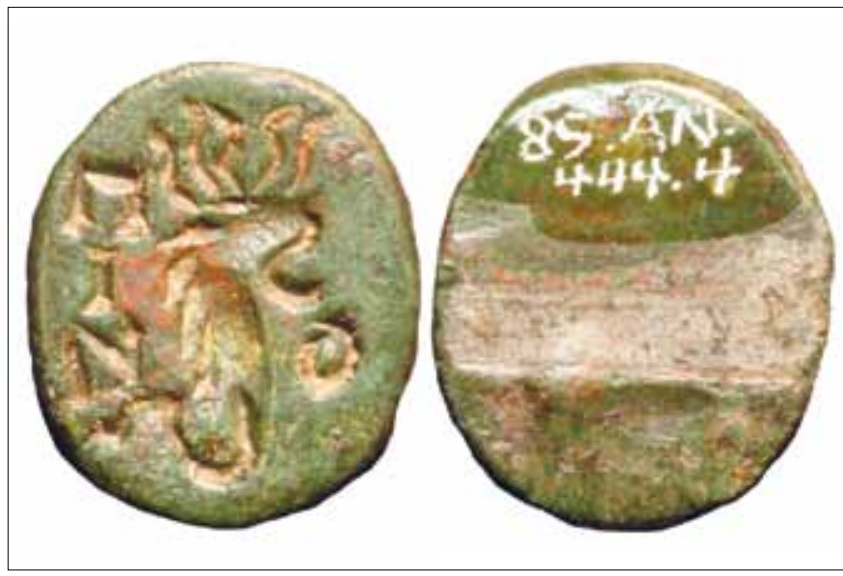

FIGURA 10. Gemma con anguipede con corona di serpenti. Malibu, Getty-85. AN 444.4 (da CBd-2390).

tuosistiche nella costruzione della scena, con la capacità di impostare volumetricamente corpi di un'organicità immaginaria, anche collocandoli in uno spazio virtuale tridimensionale.

Si hanno due esseri, con teste canine o di lupo contrapposte, volti a d., con corpo arcuato e con vello reso con una fitta tratteggiatura, che sembra anche indicare la presenza di ali. Le due bestie immaginarie hanno zampe anteriori da ungulato, mancano di quelle posteriori e hanno una sola coda anguiforme, desinente in testa di serpente, identica a quella di destra nella gemma di Londra CBd-3818 (Fig. 8)..$^{50}$

I due animali immaginari, per i quali non ho trovato finora confronti, sembrano dialogare tra loro, in una composizione piena di vivacità, con il muso della figura di destra piegato indietro verso il muso della figura di sinistra, con un effetto tridimensionale che giunge quasi alla finzione prospettica, e con le due pesanti code plasticamente attorcigliate su loro stesse..$^{51}$

Tra i segni che circondano la coppia, i due in basso e a sinistra potrebbero essere intesi come riproduzione miniaturizzata delle figure maggiori, con un effetto ideografico-geroglifico, da spiegare con il significato rituale dell'iterazione, anche ossessiva, molto frequente nelle gemme magiche, sempre proposta in termini numerici rituali. Lo si ritrova in CBd-147, a Cambridge, ${ }^{52}$ con Arpocrate sulla nave del Sole, ${ }^{53}$ con sopra le tre colonne verticali di tre volatili, tre scarabei, tre quadrupedi miniaturizzati e sotto

50 In esemplari quali quelli esaminati la qualità dell'esecuzione, la coerenza della costruzione dell'immagine, con l'utilizzo di essenziali canoni espressivi maturi, se non giunge a farci tentare il riconoscimento dell'unicità dell'artefice, ci convince comunque ad accettare l'esistenza di scuole di incisori e di centri di produzione e di formazione degli artisti.

51 In diaspro verde-nero. CBd-2129; Berlin-Aeg_s. n. Attribuita al 2-3 secolo.

52 Cambridge, Corpus Christi_C 12. Datato al 3 secolo.

53 El-Khachab 1971, 145, vi riconosce Horus sulla barca del sole. Negli amu leti del deposito Arpocrate/Horus, sulla barca, siede sempre su una sfera che deve rappresentare appunto il sole, ma che potrebbe essere anche l'utero; $\mathrm{Da}$ sen, Nagy 2019, 439-441, fig.18.8 (a p. 452). 
tre coccodrilli allineati. ${ }^{54}$ L'iterazione di immagini miniaturizzate, sempre incomprensibili per le minuscole dimensioni, è frequente nei gettoni monetali in bronzo del deposito di Cafarnao.

Pure molto vicina a CBd-2129 (Fig. 9) è la gemma CBd-2390, del Getty Museum di Malibu (Fig. 10), 55 con la rappresentazione di un simile essere fantastico, con una sola coda-serpente e con la testa ferina agitata da una capigliatura da medusa, con una raggera di otto serpenti, con a fianco la riproduzione miniaturizzata di se stesso.

Le gemme citate individuano, con altre poche presenti nel Database di Budapest, ${ }^{56}$ un nucleo, ridotto ma ben perimetrato, con scelte stilistiche e tecniche esecutive omogenee, diverse da quelle dominanti nel resto del materiale collazionato. Tutte rivelano una grande libertà nell'elaborazione e forse nella modifica delle tematiche, tanto da far quasi pensare a prodotti non seriali, più funzionali al lusso che alle pratiche magiche. Comunque sono da riferire a una dimensione ideologica e religiosa con proprie specificità, che sembra trovare, come si è detto, riferimenti nel deposito di Cafarnao.

Gli esemplari di "gemme" magiche finora esaminati, nel tentativo di inquadrare culturalmente l'amuleto n. 3766 di Cafarnao, reputo possano giustificare l'ipotesi di uno "stile della gemma CBd-3818" (Fig. 9), con prodotti di altissima qualità, funzionali alle pratiche rituali magico-religiose di una comunità ideologicamente definita, capace di esprimersi in opere d'arte figurativa con una propria tradizione artistica, maturatasi su un percorso evolutivo nel tempo che è prematuro tentare di definire, ma comunque con tecniche esecutive raffinate in grado di affrontare materiali "difficili" come le pietre dure, nelle ridotte dimensioni degli amuleti.

Le gemme del Database di Budapest indicano che si ebbe, su un arco cronologico ancora in gran parte da definire, ma comunque probabilmente esteso a tutto il 5 secolo, una produzione di costosi prodotti di lusso su pietra preziosa ${ }^{57}$ con richiesta individuale, affiancata da prodotti più modesti, monetiformi, sul tipo del nostro n. 3744, sempre individuali ma certamente con costi più accessibili e con minori possibilità di conservarsi fino ai giorni nostri.
Si può ipotizzare che a tale produzione corrispondesse a Cafarnao una qualche comunità, confraternita o "chiesa" organizzata formalmente, quale poteva essere quella ${ }^{58}$ che ci ha lasciato i testi di Nag Hamadi, scoperti nel 1945, attrezzata sicuramente per la produzione seriale a buon mercato di strumenti indispensabili per i riti magico-apotropaico-taumaturgici della setta, come i gettoni-amuleto e forse non solo. Materiali seriali, allora di scarso valore, che finora non sono stati segnalati e che forse in futuro verranno alla luce.

Il deposito di Cafarnao implica l'esistenza di un luogo fisico in cui riunirsi, che forse corrispondeva alla Sinagoga quale ora la vediamo, o essere nelle adiacenze. Possiamo comunque ipotizzare che anche altre comunità analoghe frequentassero edifici dedicati alle riunioni, alle preghiere e ai riti. ${ }^{59} \mathrm{Ne}$ rimane forse una memoria nel passo degli Acta Philippi, ${ }^{60}$ del 5 secolo, nei quali si narra come l'apostolo fosse giunto a Hierapolis e l'avesse trovata dominata dal culto dell'Echidna, la vipera, che provvide ad annientare, suscitando lo sdegno degli adoratori dei serpenti, che denunciarono come Filippo avesse "ucciso i serpenti figli della nostra dea; (i suoi fedeli) hanno chiuso il tempio e non abbiamo trovato il vino che si offre alla vipera perché ne beva e si addormenti". Filippo allora fece spalancare l'abisso, che inghiottì i fedeli dell'Echidna. ${ }^{61}$ Il riferimento al "tempio", pur nell'impostazione agiografica del racconto, appare abbastanza preciso, anche se lontano nel tempo. È probabile che il narratore ammonisse i suoi contemporanei, adoratori dei serpenti nel 4-5 secolo, con la parabola del miracolo di Filippo apostolo, datato ad età evangelica.

Sulla base di tali considerazioni ho affrontato la revisione del complesso dei documenti a noi tràditi nel deposito della cd. "Sinagoga" di Cafarnao, constatando il loro riutilizzo come gettoneamuleto.

La verifica della reale natura del deposito, che fino ad allora avevo considerato solo monetario, iniziata nell'autunno dello scorso anno 2020, si è dovuta limitare all'analisi delle scansioni effettuate a Gerusalemme nei magazzini della Custodia di Terrasanta, con apparecchiature già allora obsolete, nell'impossibilità oggi di raggiungere e manipolare direttamente il materiale, e si è immediatamente scontrata, non solo con la gestione di 23000 esemplari in sequenza fotografica casuale, ma anche con la qualità non sempre adeguata delle immagini e con l'impossibilità di effettuare analisi chimico fisiche in alcuni casi indispensabili, come si dirà più avanti.
54 Per le sequenze, di solito verticali, di animali nelle gemme magiche, cfr. Dasen, Nagy 2019, 425

55 CBd-2390, di Malibu-Getty-85.AN.444.4. La datazione proposta, il 6 secolo, e il riconoscimento improbabile come Chnoubis, indicano il disorientamento della critica nelle attribuzioni.

56 Segnalo CBd-40, con uno Chnoubis a testa leonina radiata; CBd-86, con una figura stante con asta, a testa leonina; $C B d-92$, con un babbuino accosciato circondato da animali simbolici miniaturizzati; CBd-508, con Arpocrate sul fiore di loto; CBd-543, con una figura (Arpocrate?) seduta su un coccodrillo; CBd-629, già citata sopra, con una figura stante a testa di gallo con asta, che solleva una figurina su una base; CBd-2914, con uno Chnoubis a testa leonina con raggera, ecc. Gli esemplari citati, come quelli che si aggiungerebbero con una ricerca approfondita, sono tutti più o meno stilisticamente interconnessi.

57 Dalla fine del 4 al 7 secolo le gemme magiche sarebbero presenti come oggetto di riuso; Dasen, Nagy 2019, 444-445.

\section{Cfr. già Jonas 1958, 290}

59 Nel contributo di Wilburn 2019 il tema del deposito di amuleti non viene toccato in termini utili per la situazione di Cafarnao. Non del tutto da escludere è però un'interpretazione come deposito di fondazione di un edificio.

60 Bovon, Matthews (eds.) 2012

61 D’Andria 2010, 65. Devo la preziosa segnalazione all'amico Francesco D’Andria, con l'indicazione di preziosa bibliografia e di colleghi da contattare per lo sviluppo del tema dei culti dei serpenti. Cfr. nota 27 e Filippini 2008; Albrile 2003, peri "Serpentari". 


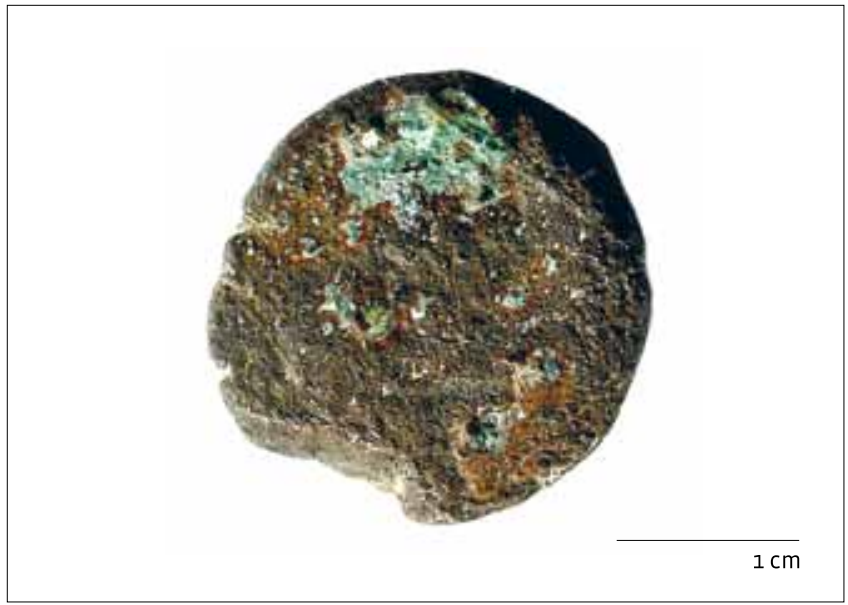

FIGURA 11. Gettone con Arpocrate sulla nave in rilievo sullo smalto verde chiaro, n. 13409, gr. 0.92 (foto E. A. Arslan).

Si è dovuto poi prendere atto del fortissimo degrado di gran parte degli esemplari, dovuto in primis al tentativo di pulizia con acidi, come già ho ricordato, intrapreso al momento dello scavo, che aveva quasi sempre, negli oltre vent'anni di permanenza dei materiali in magazzino, polverizzato le superfici, rendendo spesso illeggibili i materiali e sempre estremamente confuse le immagini, ottenute con punzoni che sembrano aver interessato percentuali altissime dei materiali recuperati.

I bagni in acido avevano infatti sempre asportato quasi completamente quanto sembrava in passato concrezione calcarea ed era invece uno strato di materia tuttora indeterminata, forse smalto, nella quale era stata data forma in rilievo a immagini diverse da quelle delle monete, coniate o fuse, riciclate come gettoni magici.

Di tali immagini rimangono solo brandelli quasi insignificanti e mai, finora, sono stati individuati tipi completamente leggibili (Fig. 11).

Molto spesso le monete risultano pulite integralmente e si può solo sospettare che vi sia stato qualche intervento per il riuso. Talvolta si hanno solo tracce impercettibili lasciate sul metallo dagli strumenti metallici utilizzati per le applicazioni.

Si può pensare, come ipotesi di lavoro da verificare con analisi, ad applicazioni di smalto, talvolta policromo. ${ }^{62}$

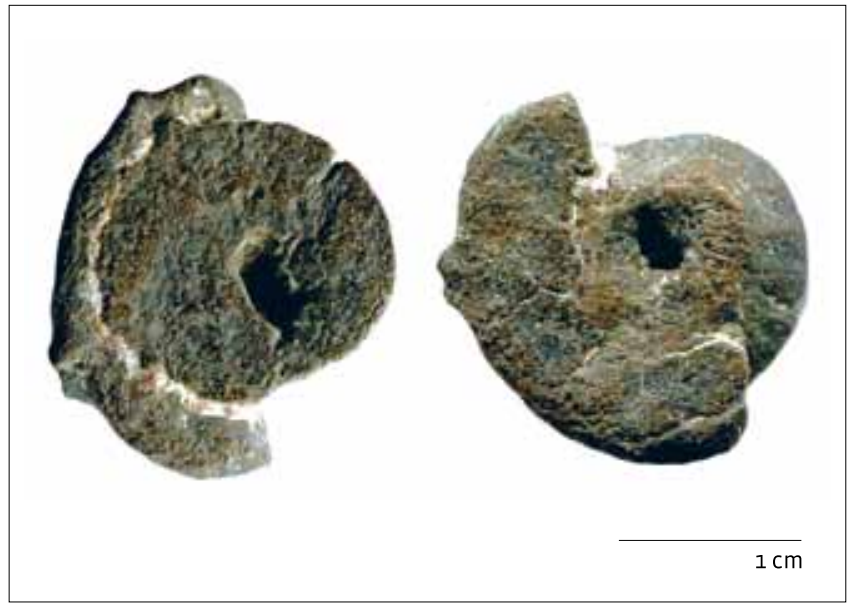

FIGURA 12. Gettone fuso con conservata parte delle matrici in argilla. Deposito n. 11006 (foto E. A. Arslan).

Accanto agli amuleti ottenuti da moneta, si registrano amuleti prodotti per fusione (Fig. 12), con tipi magico-apotropaici completi, oppure "misti", con impresso su una delle due matrici un tipo monetale, di norma il Diritto, e sull'altra matrice il tipo di un amuleto.

Non so ancora se queste tecniche sono state utilizzate contemporaneamente o se lo sono state in successione nel tempo. Una prima valutazione molto superficiale sembrerebbe confermare la produzione di gettoni amuleto con interventi ridotti sulla moneta fino all'età teodosiana e da questa e fino alla prima meta del 5 secolo di gettoni con interventi sistematici e stravolgenti sulla moneta, ${ }^{63}$ anche con applicazione di smalto. Forse dalla metà del 5 secolo si ebbe la produzione di gettoni-amuleto con immagini specifiche, ottenuti a fusione con tecnica approssimativa. La prosecuzione dell'esame della massa degli esemplari disponibili potrebbe comunque modificare queste ipotesi preliminari.

La presenza di materiale apparentemente non manipolato, talvolta discretamente conservato, non ci chiarisce ancora se la pratica dell'accumulo di amuleti, secondo rituali che è prematuro cercare di descrivere e che mi riservo di discutere in altra sede, sia stata preceduta nel tempo dal rituale dell'offerta e del getto della moneta non manipolata, ${ }^{64}$ come è avvenuto e avviene in tutte le comunità con cultura monetaria, dal passato fino ad oggi, oppure se i due rituali abbiano coesistito. ${ }^{65}$ Oppure se si è trattato del medesimo rituale modificatosi nel tempo.

\footnotetext{
62 "Lo smalto è un rivestimento inorganico fusibile che si fissa sul metallo o su ceramica ad alta temperatura (da 500 a $900{ }^{\circ} \mathrm{C}$ ). E una sostanza a base ve trosa di ogni colore, applicato spesso a oggetti di ceramica, ${ }^{[1]}$ come pure su rame, bronzo, argento e oro o loro leghe... La composizione chimica è simile a quella del vetro, in quanto formata da una miscela di silice, carbonato di sodio o potassio, Allo smalto si conferisce un aspetto opaco con l'aggiunta di stagno e altri ossidi metallici. Può declinarsi in molti colori, sfumature e finizioni" (https://it.wikipedia.org/wiki/Smalto; 08.04.2021)
}

63 Appare evidente come ci si riferisca alla produzione degli amuleti di Cafarnao e non alla datazione dei prototipi iconici dai quali derivano le immagini sui tipi. Prototipi chiaramente molto più antichi. Cfr. Zwierlein, Diehl 2019.

64 Sull'utilizzo come offerta votiva della moneta si ha ormai una sterminata bibliografia, che non è il caso di esaminare in questa sede. Cfr. il recentissimo Krmnicek, Chameroy (eds.) 2019

65 Esemplare è il caso del deposito di monete del Garigliano, nel punto di passaggio della via Appia, gettate nelle acque per ingraziarsi le divinità del fiume. Cfr. Giove 1998. 


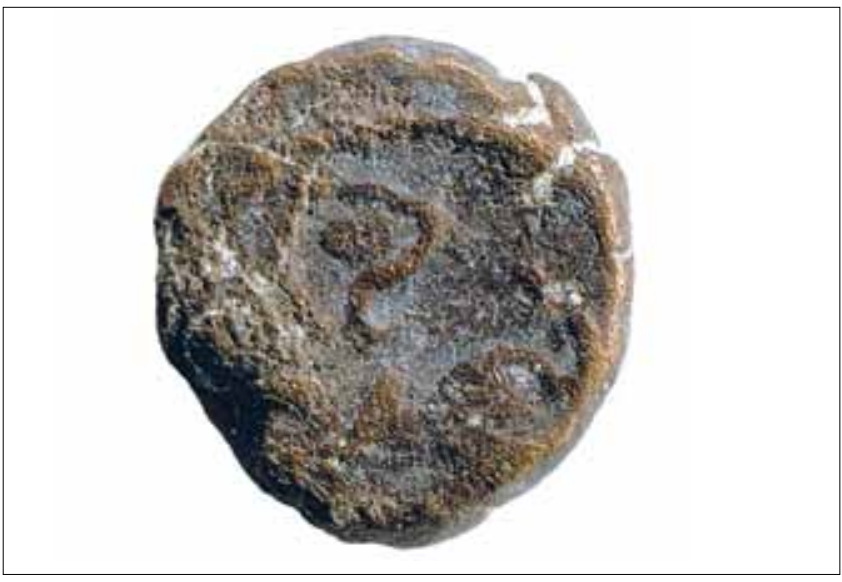

FIGURA 13. Rovescio di gettone con le serpi. Deposito n. 5869, gr. 0.96 (foto E. A. Arslan).

Infine, la presenza di esemplari monetiformi totalmente illeggibili mi ha costretto a riconoscere l'impossibilità di elaborare statistiche relative, anche se solo approssimative, alle diverse classi di materiali.

Mi devo quindi per ora limitare alla segnalazione della presenza dei pochi tipi e icone ancora riconoscibili con qualche sicurezza, quasi sempre perché presenti anche nelle gemme, dove l'incisione è sempre chiara e perfettamente leggibile. Ne è quindi possibile il riconoscimento anche da tracce minime, accostando le immagini delle gemme ai tondelli degli amuleti di Cafarnao, grazie anche al disegno spesso molto simile e alle analoghe dimensioni del supporto.

Particolarmente dolorosa è stata la difficoltà, se non l'impossibilità, ad interpretare molte punzonature o icone applicate minori, per l'eccessiva miniaturizzazione o per la cattiva conservazione del supporto. Tra queste sono probabilmente le immagini, spesso iterate in sequenza, dello scarabeo e della sua larva/Chnoubis, $^{66}$ probabilmente molto importanti nell'impaginazione dei tipi complessi, con collocazione nello specchio di una pluralità di icone, giustapposte o iterate, a comporre una "narrazione", come molto spesso si constata nelle "gemme magiche" ${ }^{67}$

Altre icone minori sono meglio riconoscibili, in quanto presenti sia miniaturizzate nelle punzonature e nelle applicazioni, sia in dimensione maggiore, occupando l'intero tipo. Tra queste abbiamo la serpe (Fig. 13) o le tre serpi, l'ape, "Abraxas" stesso, lo scarabeo ${ }^{68}$ e la sua larva/Chnubis, ${ }^{69}$ quest'ultimo presente anche solo come testa radiata (Fig. 14). Appare anche, con frequenza notevole, un'emisfera color cinabro, definita in letteratura come "utero" (Fig. 15), presente in molte gemme magiche, anche se con qualche differenza. ${ }^{70}$

Molte rimangono ancora non riconoscibili. L'esame dei 23.003 esemplari è iniziato da pochi mesi e restituisce una massa di dati ancora troppo fluida ed articolata da permettere di ospitarne le conclusioni in sedi con obbligato inevitabile contenimento dello spazio disponibile, quale quella che mi ospita in quest'occasione. Verranno suddivise nella serie di futuri contributi, pur essi preliminari, alcuni dei quali già in preparazione.

I tipi dei gettoni-amuleto e le icone delle punzonature e applicazioni sono sempre, come si è detto, di difficilissima lettura, forse anche per la scarsissima preoccupazione di proporli visibili, al momento della fabbricazione, su oggetti che acquisivano potere magico e taumaturgico per consustantazione o transustantazione..$^{71}$ Ciò veniva realizzato con l'apposizione meccanica dell'icona, seguendo certo precisi rituali, che prevedevano certo anche l'uso del gesto e della parola, ${ }^{72}$ organizzata in formule che potevano proporsi come misteriche, adeguate ad attivare la creazione dell'amuleto.

Nella realtà la nuova identità assunta da questo risultava sostanzialmente virtuale per il fedele, al quale era sufficiente anche solo la traccia dell'icona o, al limite, la certezza, se non la "fede", che la transustantazione era stata effettuata.

Da queste premesse derivava l'indifferenza per la qualità del supporto o la leggibilità delle immagini. Ciò era valido soprattutto per le punzonature che esauguravano le icone detentrici di poteri non graditi presenti sull'oggetto, per le quali era sufficiente l'azione materiale dell'applicazione.

Fondamentale per intuire le premesse ideologiche degli atti rituali nei quali erano utilizzati, dei quali potremo conoscere solo alcuni aspetti secondari grazie all'osservazione degli oggetti, appare infatti il rituale di "purificazione", al quale erano sottoposte le monete reimpiegate per diventare amuleti.

Il rituale complessivo si innestava in una tradizione, comune a tutto il mondo antico e viva ancora oggi, che prevede la consegna alla divinità di una moneta, proprio per la sua natura di oggetto con un valore preciso, del quale ci si priva intenzionalmente, per ottenere un aiuto o per ringraziare per averlo ricevuto, come ex voto.
66 Sviluppo in altra sede, nella miscellanea in memoria di Maria Teresa Grassi, in corso di elaborazione, l'ipotesi che Chnubis sia la rappresentazione della larva dello scarabeo

67 Nelle gemme magiche il campo spesso appare affollato da figure e carat teri, con notevoli analogie con le piccole stele mostrate dalle statuette amuleto, cfr. Faraone 2019

68 Per una gemma con scarabeo nel tipo, da Salonicco, montata come castone di anello, cfr. Ignatiadou 2019

69 Per il significato simbolico e religioso nell'antico Egitto dello scarabeo, come realtà animale e come immagine, cfr. Vernus, Yoyotte 2005, 441-448.
70 Per l'Utero cfr. Tsatsou 2019. Nelle gemme magiche il colore della pietra utilizzata è in rapporto con il significato delle immagini incise o all'ipotetica funzione: cfr. Masterocinque 2011; Dasen, Nagy 2019, 421, 425, 439; Amorai-Stark, Ilani 2019.

71 Non possiamo sapere, in base all'osservazione dell'amuleto, se l'entità rappresentata dall'icona si affiancasse alla realtà materiale dell'oggetto monetiforme, consustanzialmente, o se l'oggetto monetiforme assumesse, con l'apposizione dell'icona, la sua natura, substanzialmente, con esito per noi identico.

72 Sulla funzione della parola nei rituali Frankfurter 2019b 


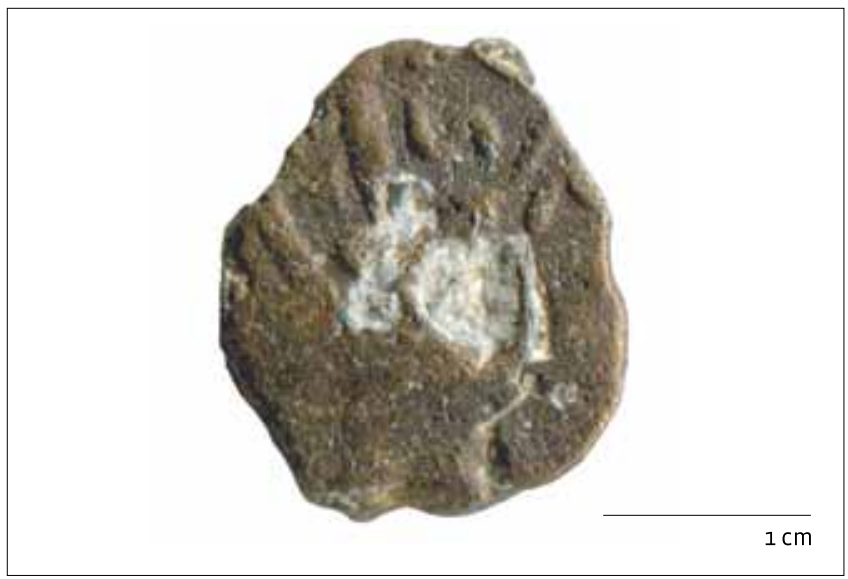

FIGURA 14. Rovescio di gettone con testa di Chnoubis radiato. Deposito n. 4240 gr. 0.94 (foto E. A. Arslan).

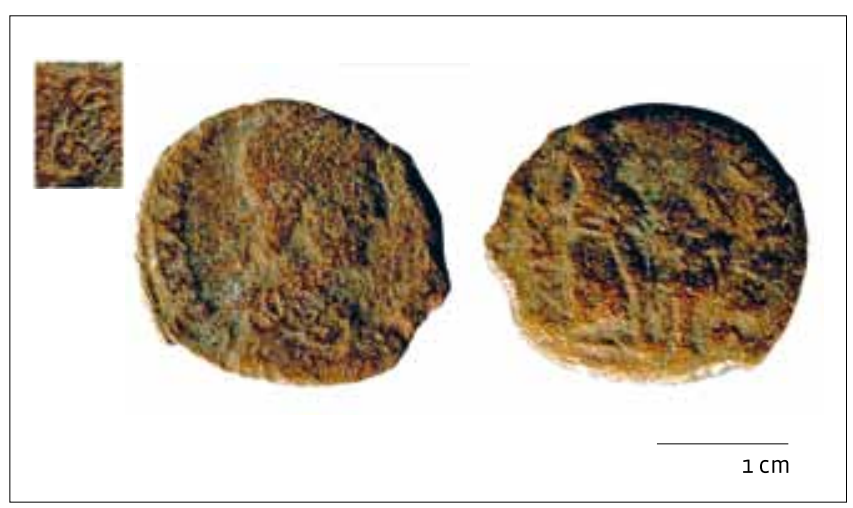

FIGURA 16. Diritto di gettone con l'imperatore con una serpe sul petto. Deposito n. 18402 , gr. 0.54 (foto E. A. Arslan).

La moneta disponibile per il rito però portava con sé, anzi “in sé”, realtà consustanziali, trasmesse al tondello con il rituale della coniazione, dal conio sigillo al tondello.

La moneta è infatti impronta sigillare, con tutte le ricadute, giuridiche e "magiche", del caso. Se sulla moneta fosse rimasto intatto il volto dell'imperatore, egli sarebbe stato virtualmente sempre presente e dalla moneta avrebbe potuto vedere, parlare, controllare, dominando l'osservatore. Tali "potenzialità" dovevano quindi essere neutralizzate, per essere sostituite dalle "potenzialità" proprie dell'amuleto, che non potevano subire interferenze. ${ }^{73}$

Quindi le immagini venivano sistematicamente, direi pedantemente, esaugurate, sia nelle monete ufficiali che in quelle imita-

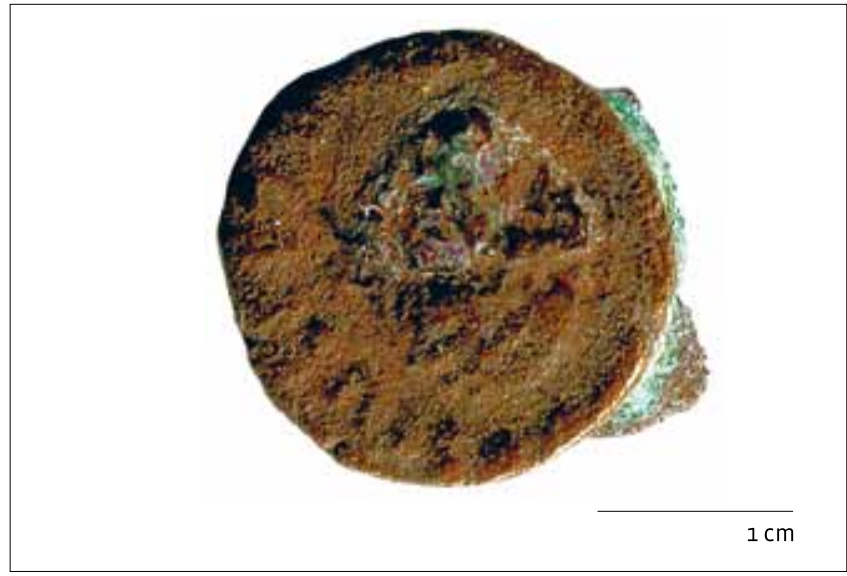

FIGURA 15. Rovescio di gettone con utero color cinabro. Deposito n. 12886, gr 0.62 (foto E. A. Arslan).

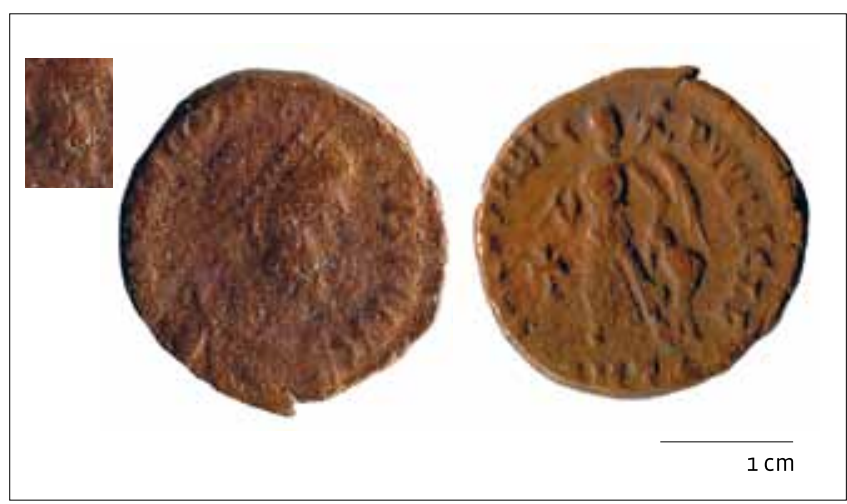

FIGURA 17. Diritto di gettone con l'imperatore con il viso cancellato. Deposito n. 19564, gr. 0.93 (foto E. A. Arslan).

tive, cancellando, con punzonature o applicazioni di smalto, gli occhi, o la bocca, o il petto (Fig. 16), o l'intero viso (Fig. 17), o il sommo del capo (Fig.18),,$^{74}$ o la cervicale. ${ }^{75}$

Nelle punzonature o applicazioni si avevano le immagini miniaturizzate delle entità destinate a sostituirsi ai poteri che venivano sottratti all'imperatore, talvolta molto specializzate.

Così al sommo del capo si collocava lo scarabeo, singolo o in sequenza, o lo Chnoubis larva miniaturizzato, a suggerire pensieri di giustizia. Così sulla bocca veniva collocata la serpe, o uno Chnoubis copriva l'intero viso, o un "Abraxas" si collocava sulla cervicale. anche in legno, sulla matrice in argilla ancora plastica, sull'impronta in negativo, prima della chiusura delle valve. Si faceva così apparire, a fusione avvenuta, l'immagine in positivo sulla superficie esterna, senza che vi fosse la cavità di norma lasciata dalla punzonatura. 


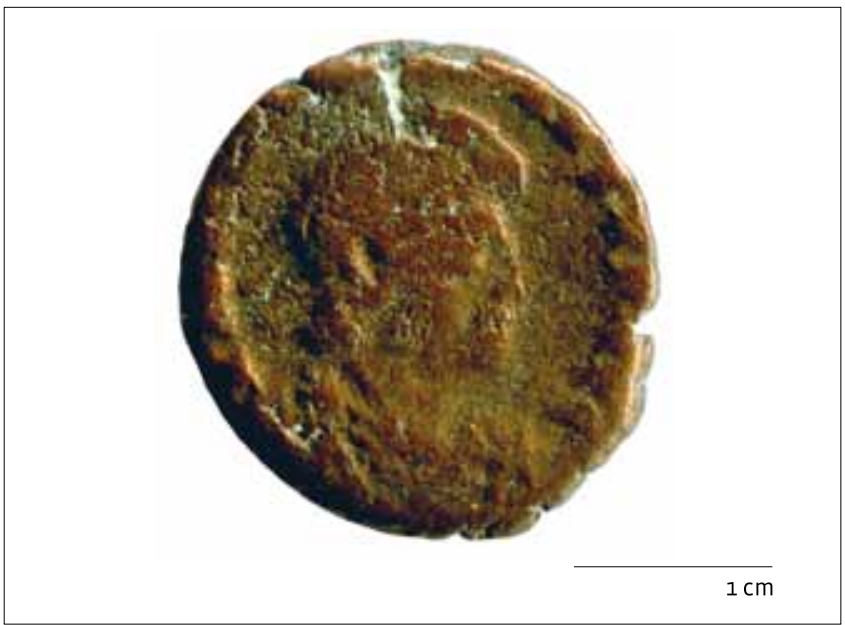

FIGURA 18. Diritto di gettone con l'imperatore con una entità al sommo del capo, forse uno Chnoubis, e con altre parti del viso cancellate. Deposito $\mathrm{n}$. 17961 , gr. 0.91 (foto E. A. Arslan)

In questi interventi, accurati ed insistiti, sicuramente affioravano e si materializzavano il disprezzo e la condanna degli Gnostici per il mondo, del quale l'imperatore rappresentava il vertice assoluto, ${ }^{76}$ che non potevano certo essere graditi all'autorità costituita. Se ne deduce la clandestinità della setta, sempre o in determinati periodi, e l'ostilità cristiana nei suoi confronti, in epoca di perfetta coincidenza di potere religioso e di potere politico-militare.

Non è forse un caso che negli interventi di esaugurazione e nei tipi degli amuleti non appaia mai alcun simbolo cristiano, con la croce dei tipi monetari reimpiegati considerata elemento ineliminabile della moneta e che quindi era priva di potenzialità negative.

In chiusura è però opportuno ancora accennare al tema dell'ambito culturale nel quale collocare la comunità presso la quale tali amuleti vennero prodotti, anche per meglio interpretare quanto finora proposto per sommi capi. Di particolare significato appare la presenza di gettoni stilisticamente e iconograficamente da collegare alle gemme nello "stile di CBd-3818".

A tale proposito appare importante il n. 4322 (Fig. 19), con una figurazione che ne sembra quasi un ricalco della figura $\mathrm{CBd}-3818$. Accettando il principio che la produzione dei gettoni-amuleto era locale, se ne deve ricavare una eccezionale resistenza nel tempo delle icone proposte e anche delle soluzioni stilistiche, anche lontano dalle datazioni di norma indicate per i prototipi, tra 1 e 3 secolo. Soprattutto risulta ora possibile individuare nell'area mediterranea orientale, in Palestina, una comunità, che prudentemente potremmo anche definire una setta "gnostica",

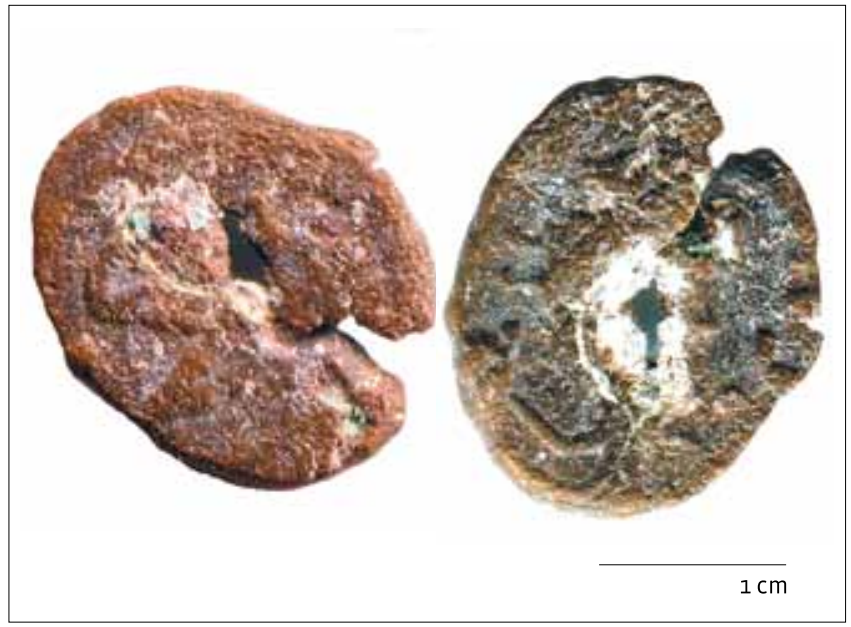

FIGURA 19. Gettone-amuleto con al D/ utero e al R/ anguipede. Deposito n. 4322 (foto E. A. Arslan).

con una propria organizzazione e forse una propria sede per il culto. Comunità che non doveva essere la sola nell'Impero, ma affiancarsi ad altre, delle quali abbiamo per ora solo indizi. Alcune con documenti che rivelano stretti collegamenti ideologici e religiosi con gli amuleti di Cafarnao, come l'amuleto di Paphos, già citato, con una simbologia che trova riferimento nella tradizione egizia, anche remota.

I materiali di Cafarnao e I'"amuleto" di Paphos, anche se certamente da intendere come "apotropaici" o "magici", non sembrano collocarsi solo nell'ambito di pratiche superstiziose ai margini dei grandi percorsi religiosi della tarda romanità, ${ }^{77} \mathrm{ma}$ anche, in termini appunto "religiosi", ${ }^{8}$ in parallelo con i culti che ebbero organizzazione ecclesiale e peso politico, il cristianesimo, il mitraismo, la religione isiaca, le correnti giudaiche, ecc. Vi è da supporre avessero una loro piena autonomia ideologica, con propri percorsi di sviluppo e con connessioni con gli altri universi religiosi, con una propria articolazione in culti e credenze diverse. Come si evince proprio dalla sintassi spesso differenziata delle icone negli amuleti-moneta di Cafarnao, nell'amuleto di Paphos o nelle diverse classi tematiche delle gemme magiche. Con la capacità di generare icone proprie, specifiche di miti e simbologie da accettare come creazioni autonome, come l'immagine di "Abraxas" o quella, pure apparentemente priva di riferimenti, della gemma di Durostorum (Moesia Inferior). ${ }^{79}$

C'è da attendersi quindi che in futuro altri complessi simili a quello di Cafarnao e altri documenti più o meno isolati vengano alla luce, imponendoci la necessità di rivedere o correggere la storia ideologica e religiosa del 5 secolo in termini finora non sospettati.
76 Per il rapporto antagonistico degli Gnostici con il mondo cfr. già Jonas 1958,254
Engemann 1975

Per il rapporto tra superstizione e magia cfr. Janowitz 2019. Appare del tutto superfluo sottolineare il labile confine tra le pratiche di culto e le pratiche superstiziose.

79 Deac, Petcu 2017 


\section{BIBLIOGRAFIA}

Albrile 2003 - E. Albrile, Il firmamento magico e l'eresia del serpente, Aspetti della mitologia gnostica nel mondo aramaico, Studi sull'Oriente Cristiano 7, 2003, 9-37.

Alexandra 2015 - Alexandra, 'The Paphos Amulet: A Reinterpretation', in Otherwise, blog, online at https://otherwiseways.wordpress.comthe-paphos-amulet-a-reinterpretation/2015/08/23/

Amorai-Stark, Ilani 2019 - S. Amorai-Stark, S. Ilani, Magical Gem Amulets from Caesarea Maritima: 'Intact' and 'Broken' Hematite Intaglios, in Endreffy, K., Nagy, A. M., Spier J. (eds.), Magical Gems in their Contexts. Proceedings of the International Workshop held at the Museum of Fine Arts, Budapest 16-18 February 2012, L'Erma di Bretschneider, 2019, 1-36.

Ancient Greek, Roman and Byzantine engraved gems in the eastern Mediterranean and Black Sea area, An international e-conference on archaeological and archaeogemological approaches, Zoom, March 11-12, 2021.

Arslan 1996a - E. A. Arslan, Una moneta alessandrina di IV secolo con Sarapis e il Nilo del lascito numismatico Carlo Rossi, Annotazioni Numismatiche 24, dicembre 1996, 538-546.

Arslan 1996b - E. A. Arslan, Il deposito di 20.323 Nummi tardo romani della Sinagoga di Cafarnao: come procedere a un campionamento scientifico, International Numismatic Newsletter 29, 1996, 6-7.

Arslan 1996c - E. A. Arslan, Monete axumite di imitazione nel deposito del cortile della Sinagoga di Cafarnao, Liber Annuus Franciscanus 46, 1996, 307-316, Pl. 17-20.

Arslan 1997 - E. A. Arslan, II deposito monetale della trincea XII nel cortile della Sinagoga di Cafarnao, Liber Annuus Franciscanus 47, 1997, 245-328.

Arslan 2003 - E. A. Arslan, Le emissioni monetarie "isiache" di IV secolo a Roma e Alessandria, in Bonacasa, N. et al. (eds.), Atti V Congresso Internazionale ItaloEgiziano, Faraoni come Dei, Tolemei come Faraoni, Torino 8-12 dicembre 2001, Museo egizio di Torino, 2003, 171-177.

Arslan 2003b - E. A. Arslan, Problemi ponderali di V secolo: verso la riforma del Nummus. Il deposito di Cafarnao, Autour de l'oeuvre numismatique de JeanPierre Callu, Journées internationales d'histoire monétaire des 20 et 21 octobre 2000, Revue Numismatique 159, 2003, 27-39.

Arslan 2011 - E. A. Arslan, The L812 Trench Deposit inside the Synagogue and the Isolated Finds of Coins in Capernaum, Israel: A Comparison of the Two Groups, Israel Numismatic Journal 6, 2011, 147-162.

Arslan 2015 - E. A. Arslan, Problemi di documentazione preliminare e finale dei ritrovamenti monetari con grandi numeri. Due esperienze: il Ripostiglio di Biassono 1975 e il "Deposito" della Sinagoga di Cafarnao (Israele), in Garraffo, S., Mazza, M. (eds.), Il tesoro di Misurata (Libia). Produzione e circolazione monetaria nell'età di Costantino il Grande, Convegno Internazionale di Studi, Roma, 10-20 aprile 2012, Edizioni del Prisma, 2015, 113-127.

Arslan 2020 - E. A. Arslan, Recensione a Chameroy/Giuhard (Hrsgg.), Produktion und Recyceln von Münzen / Produire et recycler la monnaie au Bas-Empire, 1. Internationales Numismatikertreffen / léres Rencontres internationales de Numismatique, RGZM Tagungen 29, 15-16 mai 2014, Gnomon 92, 2, 2020, 162169 .

Bonner - The Campbell Bonner Magical Gems Database-CBd, http://cbd.mfab. hu.

Bovon, Matthews (eds.) 2012 - Bovon, F., Matthews, C. R. (eds.), The Acts of Philip, A New Translation, Baylor University Press, 2012.

Callegher 1997 - B. Callegher, Un ripostiglio di monete d'oro da Cafarnao, Liber Annuus Franciscanus 47, 1997, 329-338.

Callegher 2007 - B. Callegher, Monete dall'area urbana di Cafarnao (1968-2003), Terra Santa, 2007.

Callegher 2016 - B. Callegher, Imitations and proto-vandalic nummi in the circulating stock in Upper Galilee between the end of the 5th and early 6th century: the Capernaum Deposit (locus 812), in Chameroy, J., Guihard P.-M. (eds.), Produktion und Recyceln von Münzenin der Spätantike / Produire et recycler la monnaieau Bas-empire, 1. Internationales Numismatikertreffen / 1ères Rencontres internationales de numismatique (15-16 mai 2014, Mainz), Sonderdruck / Tiré à part, RGZM - Tagungen 29, Römisch-Germanischen Zentralmuseums, 2016, 155-196.

Campedelli 2019 - C. Campedelli, Ancient Greek Magical Practices in Sicily and Southern Italy: a New Research Project, in Sánchez Natalías, C. (ed.), Litterae Magicae, Studies in Honour of Roger S. O. Tomlin, Libros Pórtico, 2019, 151-158.

Ciampini 2007 - E. M. Ciampini, Nota su un'iconografia particolare del serpente, in Mastrocinque, A. (ed.), Bollettino di Numismatica, Monografia, Sylloge Gemmarum Gnosticarum 2, Istituto poligrafico e zecca dello stato, Libreria dello stato, 2007, 213-220

Cosentino 2013 - A. Cosentino, La divinità dalla testa di gallo e gambe serpentine sulle gemme magico-gnostiche, in Baglioni, I. (ed.), Monstra, Costruzione e Percezione delle Entità Ibride e Mostruose nel Mediterraneo Antico 2 (L'Antichita Classica), Edizioni Quasar, 2013, 219-228.

D'Andria 2010 - F. D'Andria, Culti e serpenti in Anatolia, in Giancristofaro, L. (ed.), II serpente nell'area del Mediterraneo, Culti Riti Miti, Atti Convegno Internazionale, 30 aprile 2008, Cocullo, Rivista Abruzzese, 2010, 65-72.

Dasen 2019 - V. Dasen, One God May Hide Another: Magical Gems in a Cross Cultural Context, in Endreffy, K., Nagy, A. M., Spier J. (eds.), Magical Gems in their Contexts. Proceedings of the International Workshop held at the Museum of Fine Arts, Budapest 16-18 February 2012, "L'Erma" di Bretschneider, 2019, 47-58.

Dasen, Nagy 2012 - V. Dasen, Á. M. Nagy, Le serpent léontocéphale Chnoubis et la magie de l'époque romaine impériale, Anthropozoologica 47.1, 2012, 291-314.

Dasen, Nagy 2019 - V. Dasen, Á. M. Nagy, Gems, in Frankfurter, D. (ed.), Guide to the Study of Ancient Magic, Brill, 2019, 416-455.

Deac 2018 - D. Deac, Magical Gems from Porolissum, Acta Musei Porolissensis 40, 2018, 103-111.

Deac, Petcu 2017 - D. Deac, R. Petcu, A Magical Amulet from Durostorum (Moesia Inferior), in Corsten, T., Mitthof, F., Palme, B., Taeuber, H. (eds.), Tyche, Beiträge zur Alten Geschichte, Papyrologie und Epigraphik 32, Institut für Alte Ge schichte und Altertumskunde, Papyrologie und Epigraphik, Universität Wien, 2017, 8-16

Dielemann 2019 - J. Dielemann, Egypt, in Frankfurter, D. (ed.), Guide to the Study of Ancient Magic, Brill, 2019, 86-114

Di Nola 2001 - A. M. di Nola, Gli aspetti magico religiosi di una cultura subalterna italiana, Bollati Borighieri, 2001.

Doyen 2013 - J.-M. Doyen, Entre amulettes et talismans, les monnaies trouées: ce qui se cache sous les apparencesthe, Journal of Archaeological Numismatics 3, 2013, 1-37.

Eidinow 2019 - E. Eidinow, Magic and Social Tension, in Frankfurter, D. (ed.), Guide to the Study of Ancient Magic, Brill, 2019, 746-774.

El-Khachab 1971 - A. M. El-Khachab, Some Gem-Amulets Depicting Harpocrates Seated on a Lotus Flower: To the Memory of My Great Friend Dr. Alexandre Piankoff, The Journal of Egyptian Archaeology 57, 1971, 132-145.

Endreffy, Nagy 2020 - K. Endreffy, A. M. Nagy, "Apolyson apo pankakou!", A recently-surfaced magical gem, in Rocca, G., Bevilacqua, G. (eds.), Gift of a Book, Studi in memoria di David Jordan, Edizioni dell’Orso, 2020, 159-191.

Engemann 1975 - J. Engemann, Zur Verbreitung Magischer Ubelabwehr in der Nicht-Christlichen und Christlichen Spatantike, Jahrbuch fur Antike und Christentum 18, 1975, 22-48

Faraone 2019 - C. A. Faraone, Magical Gems as Miniature Amuletic Statues, in Endreffy, K., Nagy, Á. M., Spier J. (eds.), Magical Gems in their Contexts. Procee dings of the International Workshop held at the Museum of Fine Arts, Budapest 16-18 February 2012, L’Erma di Bretschneider, 2019, 85-102.

Frankfurter 2019a - D. Frankfurter, Ancient Magic in a New Key: Refining an Exotic Discipline in the History of Religions, in Frankfurter, D. (ed.), Guide to the Study of Ancient Magic, Brill, 2019, 3-26 
Frankfurter 2019b - D. Frankfurter, Spell and Speech Act: The Magic of the Spoken Word, in Frankfurter, D. (ed.), Guide to the Study of Ancient Magic, Brill 2019, $608-625$.

Frankfurter 2019c - D. Frankfurter, The Magic of Writing in Mediterranean Antiquity, in Frankfurter, D. (ed.), Guide to the Study of Ancient Magic, Brill 2019, 626-658.

Frankfurter 2019C - D. Frankfurter, Magic and the Forces of Materiality, in Frankfurter, D. (ed.), Guide to the Study of Ancient Magic, Brill, 2019, 659-677.

Giancristofaro 2010 - L. Giancristofaro, Panorami internazionali e vicissitudini di un rito, in Giancristofaro, L. (ed.), Il serpente nell'area del Mediterraneo, Culti Riti Miti, Atti Convegno Internazionale, 30 aprile 2008, Cocullo, Rivista Abruzzese, 2010, 5-9.

Giove 1998 - T. Giove, Soprintendenza Archeologica di Napoli e Caserta, Moneta del fiume Garigliano, Annali Istituto Italiano di Numismatica 45, 1998, 129-286.

Graham 2021 - L. D. Graham, The iconography on the Paphos IAEW-amulet may draw upon the apotropaic 'All-Suffering Eye' motif, Academia Letters, Article 1256, 2021, https://doi.org/10.20935/AL1256.

Ignatiadou 2019 - D. Ignatiadou, Two Magical Gems from the Roman Cemetery in Thessaloniki, in Endreffy, K., Nagy, Á. M., Spier J. (eds.), Magical Gems in their Contexts. Proceedings of the International Workshop held at the Museum of Fine Arts, Budapest 16-18 February 2012, "L'Erma” di Bretschneider, 2019, 117124.

Janowitz 2019 - N. Janowitz, The Magical Elements of Mysticism: Ritual Strategies for Encountering Divinity, in Frankfurter, D. (ed.), Guide to the Study of Ancient Magic, Brill, 2019, 678-693.

Jonas 1958 - H. Jonas, The gnostic religion: the message of the alien God and the beginnings of Christianity, Beacon Press, 1958.

Krmnicek, Chameroy (eds.) 2019 - Krmnicek, S., Chameroy, J. (eds.), Money Matters, Coin Finds and Ancient Coin Use, Habelt, 2019.

Loffreda, Cafarnao 2005 - S. Loffreda, V. Cafarnao, Documentazione fotografica degli scavi (1968-2003), Monografie dello SBF Collectio Maior, Franciscan Printing Press, 2005

Mancini 2015 - M. Mancini, Un antico amuleto con iscrizione palindroma, $A r-$ cheoMedia, Rivista di Archeologia on-line 19, 2015.

Manganaro 1963 - G. Manganaro, Nuovi documenti magici della Sicilia Orientale, Rendiconti dei Lincei 1-2, 1963, 57-70.

Mastrocinque 2003 - A. Mastrocinque, Introduzione alla Sylloge Gemmarum Gnosticarum, in Mastrocinque, A. (ed.), Bollettino di Numismatica, Monografia, Sylloge Gemmarum Gnosticarum 1, Istituto poligrafico e zecca dello stato, Libreria dello stato, 2003, 125-448

Mastrocinque 2008 - A. Mastrocinque, Un'altra immagine transculturale: Chnoubis, in Estienne, S., Jaillard, D., Lubtchansky, N. et al. (eds.), Image et Religion, Publications du Centre Jean Bérard, 2008, https://books.openedition.org/ pcjb/4620

Mastrocinque 2011 - A. Mastrocinque, The colours of magical gems, in Entwistle, C., Adams, N. (eds.), Gems of heaven, Recent research on engraved gemstones in late Antiquity C. AD 200-600, British Museum Research Publication 177, Trustees of the British Museum, 2011, 62-68.

Mastrocinque 2019 - A. Mastrocinque, Iconographies of Ialdabaōth, in Endreffy, K., Nagy, A. M., Spier J. (eds.), Magical Gems in their Contexts. Proceedings of the International Workshop held at the Museum of Fine Arts, Budapest 16-18 February 2012, “'L'Erma” di Bretschneider, 2019, 149-160

Nagy 2018 - Á. M. Nagy, L’Anguipede d’Avenches, in Bács, T. A., Bollók, A., Vida, T. (eds.), Across the Mediterranean - Along the Nile, Studies in Egyptology, Nubiology and Late Antiquity Dedicated to László Török on the Occasion of His 75th Birthday 2, Institute of Archaeology, Research Centre for the Humanities, Hungarian Academy of Sciences and Museum of Fine Arts, 2018, 939-952.

Nagy 2019 - A. M. Nagy, Figuring out the Anguipes Gems, bis: A Statistical Overview, in Endreffy, K., Nagy, Á. M., Spier J. (eds.), Magical Gems in their Contexts. Proceedings of the International Workshop held at the Museum of Fine Arts, Budapest 16-18 February 2012, "L'Erma” di Bretschneider, 2019, 179-218.

Papuci-Władyka 2011 - E. Papuci-Władyka, Z magiczną inskrypcją w języku greckim, Paphos Agora Project - nowy projektInstytutu Archeologii UJ na Cyprze, Alma Mater 139, 2011, 16-18.
Perassi 2011 - C. Perassi, Monete romane forate: qualche riflessione su "un grand thème européen" (J.-P.Callu), Aevum 85, 2, 2011, 257-315

Perassi 2011 - C. Perassi, Monete amuleto e monete talismano: fonti scritte, indizi, realia per l'età romana, Numismatica e Amtichità Classica 50, 2011, 223-274

Perea Yébenes 2018 - S. Perea Yébenes, Una gema mágica en Lisboa: la barca solar en las aguas del Inframundo, in Perea Yébenes, S., Tomás García, J. (eds.), $\lceil\lambda u \pi t o ́ s$ Glyptós Gemas y Camafeos Greco-Romanos: Arte, Mitologías, Creencias, Signifer Libros, 2018, 233-257.

Pugliese Carratelli 1953 - G. Pugliese Carratelli, Gemme magiche in Calabria, Archivio Storico Calabria e Lucania 22, 1953, 23-30.

Quack 2019 - J. F. Quack, From Egyptian Traditions to Magical Gems: Possibilities and Pitfalls in Scholarly Analysis, in Endreffy, K., Nagy, A. M., Spier J. (eds.), Magical Gems in their Contexts. Proceedings of the International Workshop held at the Museum of Fine Arts, Budapest 16-18 February 2012, "L'Erma" di Bretschneider, 2019, 233-250.

RIC 10 - J. P. C. Kent, The Roman Imperial Coinage 10, The Divided Empire and the Fall at the Western Parts 395-491, Spink, 1994.

Sliwa 2013 - J. Sliwa, Magical Amulet from Paphos with the IAEW-Palindrome, Studies in Ancient Art and Civilization 17, 2013, 293-300.

Sliwa 2015 - J. Sliwa, Amulet z Pafos z Formula Magiczna Zapisana Jako Palindrom, Meander - Rocznik Poswiecony Kulturze Swiata Starozytnego 70, 2015, $151-160$.

Sliwa 2020 - J. Sliwa, Stone Objects, in Papuci-Wladyka, E. (ed.), Paphos Agora Project (PAP) I, Interdisciplinary Research of the Jagiellonian University in Nea Paphos UNESCO World Heritage Site (2011-2015) - first results, Historia lagellonica, 2020, 423-424.

Spera 2010 - V. M. Spera, L'immagine del serpente nelle culture mediterranee, Alcuni esempi lucani e ciprioti, Il serpente nell'area del Mediterraneo, 2010, 35-64.

Spijkerman 1975 - A. Spijkerman, Cafarnao 3, Le Monete della città, Franciscan Printing Press, 1975

SGG 1 - Mastrocinque, A. (ed.), Bollettino di Numismatica, Monografia, Sylloge Gemmarum Gnosticarum 1, 2003.

SGG 2 - Mastrocinque, A. (ed.), Bollettino di Numismatica, Monografia, Sylloge Gemmarum Gnosticarum 2, 2007

Tsafrir 1995 - T. Tsafrir, The synagogues at Capernaum and Meroth and the dating of the Galilean synagogue, in The Roman and Byzantine Near East: some recent archaeological research, Supplementary series 14, Ann Arbor, MI, Journal of Roman Archaeology, 1995, 151-161 (già in Eretz Israel 20, Yadin memorial volume, Jerusalem 1989, 337-344, in ebraico. Rivisto in questa sede).

Tsatsou 2019 - E. Tsatsou, Uterine Amulets: Amulets that Protect the Uterus or that Reinforce Erotic Desire?, in Endreffy, K., Nagy, Á. M., Spier J. (eds.), Magical Gems in their Contexts. Proceedings of the International Workshop held at the Museum of Fine Arts, Budapest 16-18 February 2012, "L'Erma" di Bretschneider, 2019, 271-282.

Veiga 2007 - P. Veiga, Preliminary Study of an Unusual Graeco-Roman Magical Gem (MNA E540) in the National Museum of Archaeology in Lisbon Portugal, Conference Paper, April 2007.

Vernus, Yoyotte 2005 - P. Vernus, J. Yoyotte, Bestiaire des Pharaons, Perrin, 2005

Vitellozzi 2018 - P. Vitellozzi, Relations Between Magical Texts and Magical Gems, Recent Perspectives, De Gruyter, 2018.

Wilburn 2019 - A. T. Wilburn, Building Ritual Agency: Foundations, Floors, Doors, and Walls, in Frankfurter, D. (ed.), Guide to the Study of Ancient Magic, Brill, 2019, 555-602

Zwierlein-Diehl 2019 - E. Zwierlein-Diehl, Dating Magical Gems, in Endreffy, K., Nagy, A. M., Spier J. (eds.), Magical Gems in their Contexts. Proceedings of the International Workshop held at the Museum of Fine Arts, Budapest 16-18 February 2012, “'L'Erma” di Bretschneider, 2019, 305-338 